\title{
STELLAR KINEMATICS AND METALLICITIES IN THE ULTRA-FAINT DWARF GALAXY RETICULUM II*†*
}

J. D. Simon ${ }^{1}$, A. Drlica-Wagner ${ }^{2}$, T. S. Li ${ }^{3}$, B. Nord ${ }^{2}$, M. Geha ${ }^{4}$, K. Bechtol ${ }^{5}$, E. Balbinot ${ }^{6,7}$, E. Buckley-Geer ${ }^{2}$ H. Lin $^{2}$, J. Marshall ${ }^{3}$, B. SAntiago ${ }^{7,8}$, L. Strigari ${ }^{3}$, M. WAnG ${ }^{3}$, R. H. Wechsler ${ }^{9,10,11}$, B. Yanny ${ }^{2}$, T. Abbott ${ }^{12}$, A. H. Bauer ${ }^{13}$, G. M. Bernstein ${ }^{14}$, E. Bertin ${ }^{15,16}$, D. Brooks ${ }^{17}$, D. L. Burke ${ }^{10,11}$, D. CAPOzzi ${ }^{18}$, A. Carnero Rosell ${ }^{7,19}$, M. Carrasco Kind ${ }^{20,21}$, C. B. D’Andrea ${ }^{18}$, L. N. da Costa ${ }^{7,19}$, D. L. DePoy ${ }^{3}$, S. Desai ${ }^{22}$, H. T. Diehl ${ }^{2}$, S. Dodelson $^{2,5}$, C. E. Cunha ${ }^{10}$, J. Estrada ${ }^{2}$, A. E. Evrard ${ }^{23}$, A. Fausti Neto $^{7}$, E. Fernandez ${ }^{24}$, D. A. Finley ${ }^{2}$, B. Flaugher ${ }^{2}$, J. Frieman ${ }^{2,5}$,

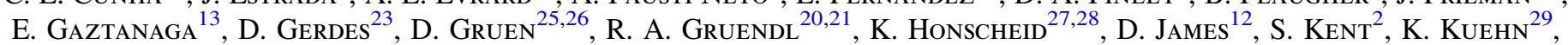

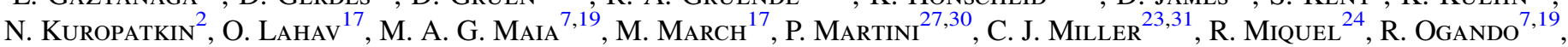
A. K. Romer $^{32}$, A. Roodman ${ }^{10,11}$, E. S. RyKoff ${ }^{10,11}$, M. SaKo ${ }^{14}$, E. SAnChez ${ }^{33}$, M. Schubnell ${ }^{23}$, I. SeVilla ${ }^{20,33}$, R. C. Smith ${ }^{12}$, M. Soares-Santos ${ }^{2}$, F. Sobreira ${ }^{2,7}$, E. Suchyta ${ }^{27,28}$, M. E. C. Swanson ${ }^{21}$, G. Tarle ${ }^{23}$, J. Thaler ${ }^{34}$, D. TuckeR ${ }^{2}$, V. Vikram $^{35}$, A. R. WALKER ${ }^{12}$, AND W. WESTER ${ }^{2}$

\author{
(The DES COLlaboration) \\ ${ }^{1}$ Carnegie Observatories, 813 Santa Barbara St., Pasadena, CA 91101, USA \\ ${ }^{2}$ Fermi National Accelerator Laboratory, P.O. Box 500, Batavia, IL 60510, USA \\ ${ }^{3}$ George P. and Cynthia Woods Mitchell Institute for Fundamental Physics and Astronomy, and Department of Physics and Astronomy, \\ Texas A\&M University, College Station, TX 77843, USA \\ ${ }^{4}$ Astronomy Department, Yale University, New Haven, CT 06520, USA \\ ${ }^{5}$ Kavli Institute for Cosmological Physics, University of Chicago, Chicago, IL 60637, USA \\ ${ }^{6}$ Department of Physics, University of Surrey, Guildford GU2 7XH, UK \\ ${ }^{7}$ Laboratório Interinstitucional de e-Astronomia-LIneA, Rua Gal. José Cristino 77, Rio de Janeiro, RJ-20921-400, Brazil \\ ${ }^{8}$ Instituto de Física, UFRGS, Caixa Postal 15051, Porto Alegre, RS-91501-970, Brazil \\ ${ }^{9}$ Department of Physics, Stanford University, 382 Via Pueblo Mall, Stanford, CA 94305, USA \\ ${ }^{10}$ Kavli Institute for Particle Astrophysics \& Cosmology, P.O. Box 2450, Stanford University, Stanford, CA 94305, USA \\ ${ }^{11}$ SLAC National Accelerator Laboratory, Menlo Park, CA 94025, USA
${ }^{12}$ Cerro Tololo Inter-American Observatory, National Optical Astronomy Observatory, Casilla 603, La Serena, Chile \\ ${ }^{13}$ Institut de Ciències de l'Espai, IEEC-CSIC, Campus UAB, Facultat de Ciències, Torre C5 par-2, E-08193 Bellaterra, Barcelona, Spain \\ ${ }^{14}$ Department of Physics and Astronomy, University of Pennsylvania, Philadelphia, PA 19104, USA \\ ${ }^{15}$ Sorbonne Universités, UPMC Univ Paris 06, UMR 7095, Institut d'Astrophysique de Paris, F-75014, Paris, France \\ ${ }_{17}^{16}$ Institut d'Astrophysique de Paris, Univ. Pierre et Marie Curie \& CNRS UMR7095, F-75014 Paris, France \\ ${ }^{17}$ Department of Physics \& Astronomy, University College London, Gower Street, London, WC1E 6BT, UK \\ ${ }^{18}$ Institute of Cosmology \& Gravitation, University of Portsmouth, Portsmouth, PO1 3FX, UK \\ ${ }^{19}$ Observatório Nacional, Rua Gal. José Cristino 77, Rio de Janeiro, RJ-20921-400, Brazil \\ ${ }^{20}$ Department of Astronomy, University of Illinois, 1002 W. Green Street, Urbana, IL 61801, USA \\ ${ }^{21}$ National Center for Supercomputing Applications, 1205 West Clark St., Urbana, IL 61801, USA \\ ${ }^{23}$ Department of Physics, University of Michigan, Ann Arbor, MI 48109, USA \\ ${ }^{24}$ Institut de Física d'Altes Energies, Universitat Autònoma de Barcelona, E-08193 Bellaterra, Barcelona, Spain \\ ${ }^{25}$ Max Planck Institute for Extraterrestrial Physics, Giessenbachstrasse, D-85748 Garching, Germany \\ ${ }^{26}$ University Observatory Munich, Scheinerstrasse 1, D-81679 Munich, Germany \\ ${ }^{27}$ Center for Cosmology and Astro-Particle Physics, The Ohio State University, Columbus, OH 43210, USA \\ ${ }^{28}$ Department of Physics, The Ohio State University, Columbus, OH 43210, USA \\ ${ }^{29}$ Australian Astronomical Observatory, North Ryde, NSW 2113, Australia \\ ${ }^{30}$ Department of Astronomy, The Ohio State University, Columbus, OH 43210, USA \\ ${ }^{31}$ Department of Astronomy, University of Michigan, Ann Arbor, MI 48109, USA \\ ${ }^{32}$ Department of Physics and Astronomy, Pevensey Building, University of Sussex, Brighton, BN1 9QH, UK \\ ${ }_{33}$ Centro de Investigaciones Energéticas, Medioambientales y Tecnológicas (CIEMAT), Madrid, Spain \\ ${ }^{34}$ Department of Astronomy, University of Illinois, 1002 W. Green Street, Urbana, IL 61801, USA \\ ${ }^{35}$ Argonne National Laboratory, 9700 South Cass Avenue, Lemont, IL 60439, USA \\ Received 2015 April 12; accepted 2015 June 16; published 2015 July 23
}

\begin{abstract}
We present Magellan/M2FS, Very Large Telescope/GIRAFFE, and Gemini South/GMOS spectroscopy of the newly discovered Milky Way satellite Reticulum II. Based on the spectra of 25 Ret II member stars selected from Dark Energy Survey imaging, we measure a mean heliocentric velocity of $62.8 \pm 0.5 \mathrm{~km} \mathrm{~s}^{-1}$ and a velocity dispersion of $3.3 \pm 0.7 \mathrm{~km} \mathrm{~s}^{-1}$. The mass-to-light ratio of Ret II within its half-light radius is $470 \pm 210 M_{\odot} / L_{\odot}$, demonstrating that it is a strongly dark matter-dominated system. Despite its spatial proximity to the Magellanic Clouds, the radial velocity of Ret II differs from that of the LMC and SMC by 199 and $83 \mathrm{~km} \mathrm{~s}^{-1}$, respectively, suggesting that it is not gravitationally bound to the Magellanic system. The likely member stars of Ret II span
\end{abstract}

\footnotetext{
* This paper includes data gathered with the $6.5 \mathrm{~m}$ Magellan Telescopes located at Las Campanas Observatory, Chile.

† Based on observations obtained at the Gemini Observatory, which is operated by the Association of Universities for Research in Astronomy, Inc., under a cooperative agreement with the NSF on behalf of the Gemini partnership: the National Science Foundation (United States), the National Research Council (Canada), CONICYT (Chile), the Australian Research Council (Australia), Ministério da Ciência, Tecnologia e Inovação (Brazil) and Ministerio de Ciencia, Tecnología e Innovación Productiva (Argentina).
}

† Based on data obtained from the ESO Science Archive Facility under request number 157689 . 
$1.3 \mathrm{dex}$ in metallicity, with a dispersion of $0.28 \pm 0.09 \mathrm{dex}$, and we identify several extremely metal-poor stars with $[\mathrm{Fe} / \mathrm{H}]<-3$. In combination with its luminosity, size, and ellipticity, these results confirm that Ret II is an ultra-faint dwarf galaxy. With a mean metallicity of $[\mathrm{Fe} / \mathrm{H}]=-2.65 \pm 0.07$, Ret II matches Segue 1 as the most metal-poor galaxy known. Although Ret II is the third-closest dwarf galaxy to the Milky Way, the line-of-sight integral of the dark matter density squared is $\log _{10}(J)=18.8 \pm 0.6 \mathrm{GeV}^{2} \mathrm{~cm}^{-5}$ within 0.2 , indicating that the predicted gamma-ray flux from dark matter annihilation in Ret II is lower than that of several other dwarf galaxies.

Key words: dark matter - galaxies: dwarf - galaxies: individual (Reticulum II) - galaxies: stellar content Local Group - stars: abundances

\section{INTRODUCTION}

The population of known dwarf galaxies orbiting the Milky Way has grown rapidly over the last decade, with the discovery of the ultra-faint dwarfs by the Sloan Digital Sky Survey (SDSS) more than doubling the size of our Galaxy's retinue of satellites (e.g., Willman et al. 2005; Zucker et al. 2006; Belokurov et al. 2007). These extreme objects are the focus of an enormous variety of ongoing work, ranging from their internal kinematics (e.g., Martin et al. 2007; Simon \& Geha 2007; Koposov et al. 2011), metallicities (e.g., Kirby et al. 2008; Norris et al. 2010), and chemical abundance patterns (e.g., Koch et al. 2008; Frebel et al. 2010, 2014; Vargas et al. 2013) to their star formation histories (e.g., Brown et al. 2014; Weisz et al. 2014a), cosmological implications (e.g., Weisz et al. 2014b), and ability to constrain dark matter models via indirect detection (e.g., Strigari et al. 2007; Ackermann et al. 2014; Geringer-Sameth et al. 2015b). However, progress has slowed in the past five years as the flow of discoveries from SDSS dwindled (although see Kim et al. 2015; Laevens et al. 2015; and Martin et al. 2015).

The recent discovery of eight new candidate dwarf galaxies (Bechtol et al. 2015; Koposov et al. 2015) in $2000 \mathrm{deg}^{2}$ of DECam imaging data from the first year of the Dark Energy Survey (DES; Diehl 2014; Flaugher et al. 2015) promises to reinvigorate studies of the faintest galaxies. Some of the particularly interesting aspects of these newly discovered objects are their apparent concentration around the Magellanic Clouds, the identification of a relatively luminous dwarf (Eridanus II) near the Milky Way's virial radius with possible recent star formation, and the existence of hyper-faint $\left(M_{V} \lesssim-3\right)$ dwarfs beyond the immediate vicinity of the Milky Way. Four of these new satellites-Reticulum II, Eridanus II, Tucana II, and Horologium I-can be fairly confidently classified as galaxies based on DES imaging alone, while the other systems have physical sizes and luminosities that overlap within their uncertainties with those of some globular clusters.

In this paper we begin the process of spectroscopic followup observations of the new DES ultra-faint satellites. We present low and high resolution spectroscopy of stars in Ret II, the closest $(d=32 \mathrm{kpc})$ and best-characterized of the DES satellites, with an ellipticity of 0.6 and a projected elliptical half-light radius of $0: 1$ (Bechtol et al. 2015). The velocities and metallicities of these stars confirm that Ret II is indeed a dwarf galaxy. In Section 2 we describe our spectroscopic target selection, observations, and data reduction. We discuss the measurement of stellar velocities and metallicities and the classification of Ret II member stars in Section 3. We focus on some of the implications of our results and examine how Ret II fits into the previously known population of Milky Way satellite galaxies in Section 4. In Section 5 we summarize our findings and conclude.

\section{OBSERVATIONS AND DATA REDUCTION}

\subsection{Target Selection}

Spectroscopic targets were selected from the object catalog derived from the coadded images of the first internal annual release of DES data (Y1A1; Sevilla et al. 2011; Desai et al. 2012; Mohr et al. 2012; Balbinot et al. 2015; R. A. Gruendl et al., 2015 in preparation). We identified objects as stars based on the spread_model quantity output by SExtractor (Bertin 2011; Desai et al. 2012). Our stellar sample consists of well-measured objects with $\mid$ spread_model_i $\mid<0.002$ and flags_\{ $g, r, i\}<4$. We selected likely members based on a matched-filter maximum likelihood procedure combining a spatial model of Ret II and the predicted color-magnitude distribution of an old metal-poor stellar population (Bechtol et al. 2015). We modeled the spatial distribution of Ret II with the best-fit elliptical Plummer profile with ellipticity of 0.6 and an elliptical half-light radius of 0.1 . The distribution of stars in color-magnitude space was modeled by a composite of four isochrones bracketing a range of ages, $\tau=\{12.6,14.1 \mathrm{Gyr}\}$, and metallicities, $Z=\{0.0001,0.0002\}$ (Marigo et al. 2008; Girardi et al. 2010). These isochrones are placed at the best-fit distance modulus of $m-M=17.5$ and weighted by the initial mass function of Chabrier (2001). We used a composite isochrone to maximize the number of candidate members while remaining agnostic to the exact age and metallicity of Ret II.

One result of the maximum-likelihood fit is a membership probability for each object in our stellar catalog (Rozo et al. 2009; Bechtol et al. 2015). This membership probability incorporates both the spatial separation of the star from the centroid of Ret II and the distance of the object from the composite isochrone in color-magnitude space. Our spectroscopic follow-up sample primarily consisted of stars with membership probability $p>0.01$ within 0.5 of the centroid of Ret II. Each of these objects was visually inspected in the DES coadded images and the imaging was confirmed to be of high quality.

We targeted Ret II for follow-up spectroscopy with both Magellan/M2FS and Gemini/GMOS, and we have also made use of publicly available Very Large Telescope (VLT)/ GIRAFFE spectra of Ret II stars in the ESO Archive. For observations with the 256-fiber Magellan/M2FS spectrograph (Section 2.2), we targeted objects within 14! 65 of Ret II with $p \geqslant 0.01$ and $g \leqslant 22$. A small fraction of the 177 targets meeting these criteria were not observed because of fiber collisions and exclusion regions around bright stars. Given the availability of additional fibers, we also added 18 stars located near the Ret II isochrone that missed the probability and/or photometric quality cuts. Out of these 195 stars, a total of 185 were targeted with science fibers. Six fibers were broken, and the remaining 65 were placed on blank sky positions chosen to have low count rates from a Digitized Sky Survey image. 
The Gemini/GMOS observation field (Section 2.3) was selected as the $5^{\prime} \times 5^{\prime}$ region with the highest summed membership probability. Slits were iteratively prioritized based on the membership probability and brightness of targets, focusing on stars on the giant branch for which metallicities could be measured via the $\mathrm{Ca}$ triplet $(\mathrm{CaT})$ lines. Final mask creation was performed using the GMMPS v0.402 toolkit, which was used to assign slits to 33 science targets and 4 acquisition objects. ${ }^{36}$

VLT/GIRAFFE observations of Ret II (Section 2.4) were carried out under the auspices of the Gaia/ESO Survey (Gilmore et al. 2012), and the target selection was performed by the Gaia/ESO Survey team independently of our DES photometric analysis chain. It is clear from the colors and magnitudes of the observed stars that they were chosen to lie near the Ret II fiducial sequence determined by Koposov et al. (2015), but we do not have access to the specific criteria by which priorities were set. Comparing this sample with our catalog, we find that 14 of the stars observed are highprobability member candidates $(p>0.5), 8$ have low to intermediate membership probabilities $(0.5>p>0.01)$, and 23 are unlikely to be members $(p<0.01)$. The majority $(62)$ of the VLT spectra are of bright stars not included in the DES coadd catalog because of its saturation limit at $g \sim 17$, but (with one exception; Section 3.3.2) these stars appear to have been selected to be Milky Way disk stars and are not relevant for our purposes.

\subsection{Magellan/M2FS Spectra}

We observed Ret II with the multi-fiber M2FS spectrograph (Mateo et al. 2012) on the Magellan/Clay Telescope on 2015 February 19. M2FS consists of two identical spectrographs, generally referred to as "red" and "blue" even though neither is optimized for a particular wavelength range. Each spectrograph is fed by 1281 ". 2 fibers, which are positioned on the sky over a 29 ! 5 diameter field with a plug plate. We used M2FS in its high resolution configuration at $R \approx 25000$, with a narrow-band filter to isolate a single spectral order covering the $\mathrm{Mg}$ b spectral region (5120-5190 $\AA$ ) for each fiber. The 256 M2FS fibers were placed on 185 stars selected from the DES Y1A1 internal data release, as well as 65 blank sky positions for sky subtraction, as described in Section 2.1.

We obtained three $2400 \mathrm{~s}$ exposures on the Ret II field in variable seeing conditions and with decreased transparency resulting from thin clouds. We also obtained twilight sky spectra for flatfielding, ThArNe lamp frames for wavelength calibration, and spectra of the $\mathrm{K}$ giant radial velocity standard star CD $-43^{\circ} 2527$ through a single fiber to check the velocity zero point.

We reduced the data with the dohydra package in IRAF. We began by subtracting the bias level, recombining the files from the four amplifiers used to read out each CCD into a single frame, and masking out cosmic rays using the L.A. Cosmic routine (van Dokkum 2001). We then trimmed the frames to the central 1000 pixels to eliminate contamination from neighboring spectral orders. We traced the fiber positions as a function of wavelength using a twilight frame and removed scattered light with a two-dimensional fit to the areas between each group of 16 fibers. Because of the low signal-to-noise ratio $(\mathrm{S} / \mathrm{N})$ of the data (the brightest stars reach $\mathrm{S} / \mathrm{N} \approx 20$ pixel $^{-1}$, and the large majority

\footnotetext{
36 http://www.gemini.edu/node/12255?q=node/10458
}

of the targets are at $\mathrm{S} / \mathrm{N}<10$ pixel $^{-1}$ ) and the excellent flatness of the $\mathrm{e} 2 \mathrm{~V}$ detectors $(\sim 1 \%)$, we did not attempt to apply a flatfield correction (Massey \& Hanson 2013). We extracted the spectrum of each fiber in the ThArNe lamp, twilight, standard star, and science frames. We also performed the same extraction on a variance image derived from the science frames to provide spectra of the pixel uncertainties. We determined the wavelength solution by fitting a fourth order polynomial to known Th and Ar line wavelengths on the ThArNe exposures. The positions of the ThAr lines shifted by one pixel $\left(3.8 \mathrm{~km} \mathrm{~s}^{-1}\right)$ in the wavelength direction between the ThArNe frame taken immediately before Ret II was observed and the one taken immediately after. By examining the absorption lines of the highest $\mathrm{S} / \mathrm{N}$ stars, we determined that in the blue spectrograph, this shift occurred between the second and third Ret II exposures. In the red spectrograph all three Ret II frames appeared to be aligned with each other. We therefore shifted the third frame taken with the blue spectrograph by one pixel and re-ran the reductions using only the ThArNe frame from before the Ret II observing sequence (to maintain consistency between the velocity scales for the red and blue spectrographs, the wavelength solution for the red data was also determined with the ThArNe frame observed before Ret II). This wavelength solution was then applied to the data and the spectra were rebinned. We constructed master sky spectra for the red and blue spectrographs by coadding the $\sim 30$ sky fiber spectra obtained in each spectrograph. The sky spectra were scaled according to the throughput of each target fiber (determined from the twilight sky frames) and subtracted.

\subsection{Gemini/GMOS Spectra}

We also observed Ret II with the GMOS-S spectrograph (Hook \& Allington-Smith 2004) on the Gemini South Telescope in queue mode on several nights beginning on 2015 February 15 through program GS-2014B-DD-8. We targeted 33 likely Ret II member stars with a multi-slit mask, using $0^{\prime \prime} .75$ slits and a minimum slit length of $5^{\prime \prime}$. Our observations employed the R831 grating to produce a spectral resolution of $R=4400$ over a range of $2300 \AA$ per spectrum, and the RG610 filter to block second order light. A total of eleven $1200 \mathrm{~s}$ science-quality exposures were obtained. We observed at two different central wavelengths around the nearinfrared CaT lines $(8550$ and $8650 \AA)$ to ensure that key spectral features did not land in gaps between the three CCDs. Science observations were alternated with Quartz-Halogen flats and $\mathrm{CuAr}$ arc lamp exposures for calibration.

We reduced the GMOS data using version 2.16 of the Gemini package in IRAF. For each dither on each night of observations, we created a flat field frame and used it to process the science frame $(\mathrm{s})$. We combined exposures from the same dithers and then derived the wavelength solution. The spectra were rectified and transformed onto a common wavelength scale, and finally the dithers were coadded to eliminate the CCD gaps. We performed sky subtraction on each slit by masking out the stellar continuum and fitting a linear function to the background in the spatial direction. Extractions were carried out on the sky-subtracted spectra.

\subsection{VLT/GIRAFFE Spectra}

Ret II was observed with the FLAMES/GIRAFFE spectrograph (Pasquini et al. 2000) on the VLT/UT2 telescope 
as part of the Gaia/ESO survey (Gilmore et al. 2012). Because the Gaia/ESO survey is a public spectroscopic survey, these data are public and available from the ESO Archive ${ }^{37}$ immediately after the observations are obtained.

Observations were taken in MEDUSA mode, which allows the simultaneous observation of up to 132 objects. On the night of 2015 March 9, $2 \times 1500 \mathrm{~s}$ exposures were taken using the HR21 grating, which covers the wavelength range from 8482-8981 $\AA$ at a resolution of $R \sim 16200$. The same field was observed with a bluer wavelength setting in 2015 February, but because of the lower S/N we do not make use of those data in this paper. The calibration frames taken as part of the Gaia/ESO Survey observations include 5 biases, 3 flats, and $1 \mathrm{ThAr}$ arc taken at the end of the night. We reduced the data with the GIRAFFE Gasgano pipeline (v2.4.8) provided by ESO. ${ }^{38}$ This pipeline provides utilities for bias subtraction, flat-fielding, spectral extraction of individual objects and accurate wavelength calibration.

The CaT absorption lines are located close to a number of bright sky emission lines, so we require accurate sky subtraction to recover their positions and equivalent widths (EWs). We first determined fiber to fiber wavelength offsets for the 16 GIRAFFE sky fibers using the night sky lines, finding that there are shifts of $\sim 0.15 \mathrm{~km} \mathrm{~s}^{-1}$ between fibers. We then shifted the sky spectra based on these calculated offsets, linearly interpolated them, and combined them into a master sky spectrum, rejecting outliers with a $3 \sigma$ clip. For each science spectrum, we determined the wavelength offset relative to the master sky and shifted the data accordingly. We scaled the sky spectrum to match the amplitude of the bright, isolated sky lines at 8885.85 and $8919.6 \AA$ and subtracted it from the science spectrum.

\section{VELOCITY AND METALLICITY MEASUREMENTS}

Because the M2FS spectra have higher spectral resolution than the other data sets and cover the largest sample of candidate Ret II member stars, they are particularly well suited for measuring the stellar kinematics of Ret II. The GIRAFFE spectra could also be used for accurate radial velocity measurements (e.g., Koposov et al. 2011), but they include a smaller number of likely Ret II members, nearly all of which overlap with the M2FS target list. In order to avoid the complications of placing multiple instruments on a consistent velocity scale, we choose to use M2FS alone for the primary kinematic results reported in this paper. However, as we note in Section 4.1, we obtain consistent results with the GIRAFFE velocities. The GMOS observations were made at much lower spectral resolution and would not be competitive with M2FS or GIRAFFE for velocity work. GIRAFFE and GMOS velocities for stars not observed with M2FS are listed in Table 1 but are not used to constrain the velocity dispersion of Ret II.

The wavelength range covered by the M2FS observations is not well-suited to metallicity measurements using the spectra alone, because the strength of the $\mathrm{Mg} \mathrm{b}$ triplet lines depends on a number of stellar properties: effective temperature, surface gravity, metallicity, and $\mathrm{Mg}$ abundance. No calibrations for the metallicities of individual stars as a function of $\mathrm{Mg}$ b strength are available in the literature. We therefore rely on the better-

\footnotetext{
37 http://archive.eso.org/cms/eso-data.html

38 http://www.eso.org/sci/software/gasgano.html
}

studied CaT region of the spectrum in the VLT and Gemini data for metallicity information.

The primary data used in our analysis for the remainder of the paper are 52 M2FS velocity measurements, 16 GIRAFFE metallicities, and 6 GMOS metallicities. All velocity measurements presented here have been transformed to the heliocentric frame. We use heliocentric corrections of $-8.26 \mathrm{~km} \mathrm{~s}^{-1}$ for the M2FS observations and $-5.63 \mathrm{~km} \mathrm{~s}^{-1}$ for the GIRAFFE observations.

\subsection{Radial Velocity Measurements}

Given a velocity data set in which the intrinsic velocity dispersion may not be much larger than the uncertainties on individual velocity measurements, accurate characterization of both the velocities and the measurement uncertainties is critical for a reliable determination of the velocity dispersion. We used Monte Carlo simulations to investigate the statistical uncertainties of our velocity measurements, and repeat observations to constrain systematic uncertainties.

We determined radial velocities using the same basic approach as in Simon \& Geha (2007) and subsequent papers. We performed a $\chi^{2}$ fit to each spectrum with a high $\mathrm{S} / \mathrm{N}$ template spectrum as a function of the velocity offset between the two (Adelman-McCarthy et al. 2008; Lee et al. 2008; Newman et al. 2013). As a template spectrum for this analysis, we chose a high resolution spectrum of the metal-poor giant HD 122563 obtained in 2009 with Magellan/MIKE, because the M2FS RV standard CD-43 2527 is relatively metal-rich and does not provide a very good match to the spectra of the Ret II stars. We assume a heliocentric velocity of $-26.5 \mathrm{~km} \mathrm{~s}^{-1}$ for HD 122563 (Chubak et al. 2012). We then ran 500 Monte Carlo simulations per star, in which noise comparable to the calculated variance in each pixel was added to the spectrum and the velocity was re-measured as above. The statistical uncertainty on the velocity of each star was defined to be the square root of the variance in the measured mean velocity for the 500 Monte Carlo spectra. Highly discrepant Monte Carlo iterations were discarded before computing the variance. The median statistical uncertainty on the velocity measurements for the M2FS data set is $1.0 \mathrm{~km} \mathrm{~s}^{-1}$.

As a test of systematic velocity uncertainties in the M2FS data, we reduced the three Ret II science frames separately, and measured velocities for the brightest stars in each frame. Despite carrying out these reductions in multiple ways and computing velocities with two different techniques, we found that the velocity difference between independent measurements of a star on frames $i$ and $j, \Delta v=v_{i}-v_{j}$, is consistently larger than would be expected from the statistical uncertainties on each individual velocity measurement $\left(v_{\mathrm{err}}=\sqrt{v_{\mathrm{err}, i}^{2}+v_{\mathrm{err}, j}^{2}}\right)$. To obtain a standard deviation of $\Delta v / v_{\text {err }}$ of one, we needed to add an additional $0.9 \mathrm{~km} \mathrm{~s}^{-1}$ uncertainty in quadrature with $v_{\mathrm{err}}$. We consider this value of $0.9 \mathrm{~km} \mathrm{~s}^{-1}$ to be the systematic uncertainty in the M2FS velocity measurements, and we define the total velocity uncertainty for each spectrum to be the quadrature sum of the Monte Carlo uncertainty from the template fit and the systematic uncertainty. A separate check on the systematics and their possible origin is provided by the high S/N M2FS twilight sky spectra. We fit these twilight spectra with a high resolution solar template spectrum. The scatter in velocity from fiber to fiber was $\leqslant 0.20 \mathrm{~km} \mathrm{~s}^{-1}$, so we conclude that the internal velocity errors over short timescales on an 
individual frame (incorporating, e.g., any fiber-to-fiber systematics) are negligible. However, over multiple science exposures spanning several hours, this is not necessarily the case (see above).

In order to verify the reliability of our velocity zero point, we also measured the velocity of the radial velocity standard star CD $-43^{\circ} 2527$ by fitting it with the HD 122563 template, exactly as we did for the science spectra. For the two exposures on $\mathrm{CD}-43^{\circ} 2527$, we find $v_{\text {hel }}=19.6 \pm 0.1$ $\mathrm{km} \mathrm{s}^{-1}$ and $v_{\text {hel }}=19.9 \pm 0.1 \mathrm{~km} \mathrm{~s}^{-1}$, compared to the cataloged velocity of $v_{\text {hel }}=19.7 \pm 0.9 \mathrm{~km} \mathrm{~s}^{-1}$ (Udry et al. 1999).

\subsection{Metallicity Measurements}

We calculated metallicities for 16 Ret II RGB stars with the CaT calibration of Carrera et al. (2013). As recommended by Hendricks et al. (2014), we measured the EWs of the CaT lines in the same way as Carrera et al., fitting each of the three lines with a Gaussian plus Lorentzian profile. Also following Carrera et al. (2013), we adopt the line and continuum regions defined by Cenarro et al. (2001), except for the 8498 $\AA$ line. Cenarro et al. employed a continuum bandpass of 8474-8484 $\AA$ for this line, but the blue limit of the GIRAFFE spectra is $8482 \AA$, so we instead use a region on the red side of the line from $8513-8522 \AA$. This wavelength range may be modestly affected by two weak Fe I lines at 8514 and $8515 \AA$, but at the metallicity of typical ultra-faint dwarf stars any resulting depression of the continuum should be negligible over a $9 \AA$ A band.

CaT metallicity measurements usually use the horizontal branch (HB) magnitude to correct for the dependence of the CaT EWs on stellar luminosity. The HB magnitude of Ret II, however, is not well determined because the galaxy contains so few HB stars. We therefore rely on the calibration of CaT EW as a function of absolute $V$ magnitude from Carrera et al. (2013). We convert the DES $g$ and $r$ magnitudes to the SDSS photometric system, and then use the relations for metal-poor stars from Jordi et al. (2006) to transform to $V$. We determine absolute magnitudes assuming a distance of $32 \pm 3 \mathrm{kpc}$ (Bechtol et al. 2015) and a $V$-band extinction of $A_{V}=0.05$ mag (Schlafly \& Finkbeiner 2011).

\subsection{Spectroscopic Membership Determination}

\subsection{1. $M 2 F S$}

Out of the 185 M2FS fibers placed on stars, we successfully measured velocities for 52, including a large majority of the observed targets brighter than $g=20.6$. The remaining stars had $\mathrm{S} / \mathrm{N}$ ratios too low for spectral features to be confidently detected in the data. The velocity measurements and other properties of the stars are listed in Table 1. The velocity distribution we measure from the M2FS spectra exhibits a strong peak at a velocity of $\sim 60 \mathrm{~km} \mathrm{~s}^{-1}$ (see Figure 1), as is characteristic of a gravitationally bound system. Approximately half of the stars for which we measure velocities are contained in this peak, with the remainder spread across a wide range of heliocentric velocities from $\sim 0$ to $\sim 330 \mathrm{~km} \mathrm{~s}^{-1}$.

For a large majority of the observed stars, the membership status is unambiguous; stars with $v_{\text {hel }}>90 \mathrm{~km} \mathrm{~s}^{-1}$ and $v_{\text {hel }}<40 \mathrm{~km} \mathrm{~s}^{-1}$ are clearly not related to the peak associated with Ret II, while those very near the mean velocity of the system and close to the central position spatially are almost certainly members. However, to ensure that the member sample is defined optimally we carefully examine all stars within $20 \mathrm{~km} \mathrm{~s}^{-1}$ of the mean velocity of Ret II, considering their velocities, positions in the color-magnitude diagram, spatial locations, membership probabilities from Bechtol et al. (2015), and spectral features. Below we discuss the individual stars whose membership is not immediately obvious.

Three stars in our sample have velocities of $v_{\text {hel }} \sim 50 \mathrm{~km} \mathrm{~s}^{-1}$, just to the left of the Ret II peak in Figure 1(c), and about $15 \mathrm{~km} \mathrm{~s}^{-1}$ away from the systemic velocity. Of these three, DES J033405.49-540349.9 is very metal-rich, DES J033437.34 -535354.0 is a double-lined spectroscopic binary with two strong cross-correlation peaks separated by $\sim 60 \mathrm{~km} \mathrm{~s}^{-1}$, and DES J033540.70-541005.1 is located close to two half-light radii away from the center of Ret II along the minor axis and is separated from the mean velocity by $\sim 4 \sigma$. None of these stars has characteristics consistent with membership in Ret II. DES J033540.70-541005.1 has colors consistent with what would be expected for a red HB star in Ret II, but is $\sim 0.1$ mag fainter than the isochrone shown in Figure 1(a). Given its spatial and velocity offsets from Ret II as well as its position in the color-magnitude diagram, it is almost certainly not a member. Spectra of these three non-member stars are displayed in Figure 2.

Another group of four stars is located slightly to the blue of the isochrones we use to describe the Ret II stellar population (by $\sim 0.02-0.06 \mathrm{mag}$ ). DES J033524.00-540226.7 is the farthest from the isochrone, but is also the faintest of these stars (sitting near the base of the red giant branch) and consequently has the largest photometric uncertainties. Its velocity is within $3 \mathrm{~km} \mathrm{~s}^{-1}$ of the systemic velocity of Ret II and it is located inside the half-light radius, so we consider it a member. DES J033635.78-540120.2 is also relatively faint, but is farther away from Ret II in both position $\left(\right.$ at $\sim 1.3 R_{1 / 2}$ ) and velocity $\left(6 \mathrm{~km} \mathrm{~s}^{-1}\right)$. It is likely a member of Ret II, but the spatial and velocity offsets make that classification less certain. DES J033531.14-540148.3 is one of the brightest candidate members, in the clump of stars near $g \sim 17.5$ in Figure 1(a). It is within the half-light radius of Ret II and has a very low metallicity as determined from the GIRAFFE CaT measurements. Because of its low metallicity and spatial position, we conclude that it is a Ret II member. The color offset to the blue of the giant branch could indicate that it is an asymptotic giant branch star. DES J033550.10-540139.2 is located quite close to the center of Ret II, but is one of the bluest (non-HB) stars with a velocity consistent with that of Ret II. This star is projected quite close to the center of Ret II and has extremely weak absorption lines. If it is indeed a member, it could have an unusually low metallicity. As a result of the weak spectral features, the velocity is quite uncertain; it is consistent with membership, but it has little impact on the derived properties of Ret II because of the large uncertainty.

Two stars are offset to the red side of the isochrone. DES J033436.70-540645.0 is almost two half-light radii from the center of Ret II, although it is very close to the position of the velocity peak. It also has strong absorption lines, indicating a relatively high metallicity. Given that it is offset from the rest of the Ret II population in color, spatial position, and metallicity, we conclude that it is probably not a member. DES J033544.18-540150.0 is at the centroid of the Ret II velocity peak within the uncertainties, and is also located very close to the central position, but is $\sim 0.04$ mag redder than the 

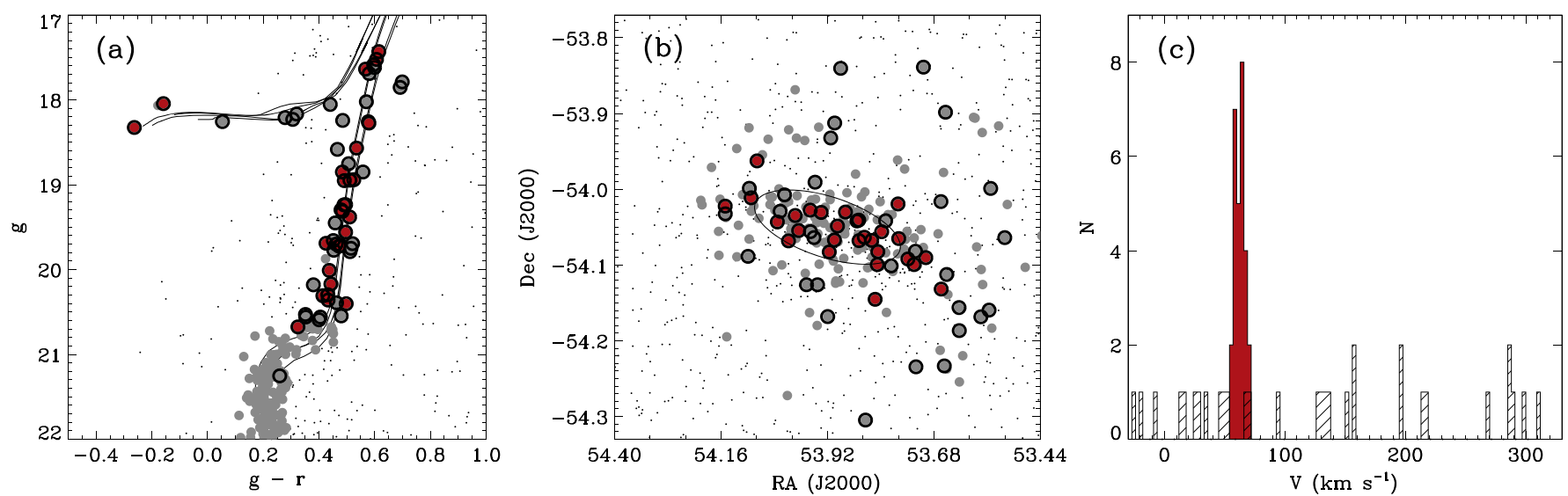

Figure 1. (a) DES color-magnitude diagram of Reticulum II. Stars within 14! 65 of the center of Ret II are plotted as small black dots, and stars selected for spectroscopy with M2FS, GIRAFFE, and GMOS (as described in Section 2.1) are plotted as filled gray circles. Points surrounded by black outlines represent the stars for which we obtained successful velocity measurements, and those we identify as Ret II members are filled in with red. The four PARSEC isochrones used to determine membership probabilities are displayed as black lines. (b) Spatial distribution of the observed stars. Symbols are as in panel (a). The half-light radius of Ret II from Bechtol et al. (2015) is outlined as a black ellipse. (c) Radial velocity distribution of observed stars, combining all three spectroscopic data sets. The clear narrow peak of stars at $v \sim 60 \mathrm{~km} \mathrm{~s}^{-1}$ highlighted in red is the signature of Ret II. The hatched histogram indicates stars that are not members of Ret II; note that there are two bins containing non-member stars near $v=70 \mathrm{~km} \mathrm{~s}^{-1}$ that are over-plotted on top of the red histogram.

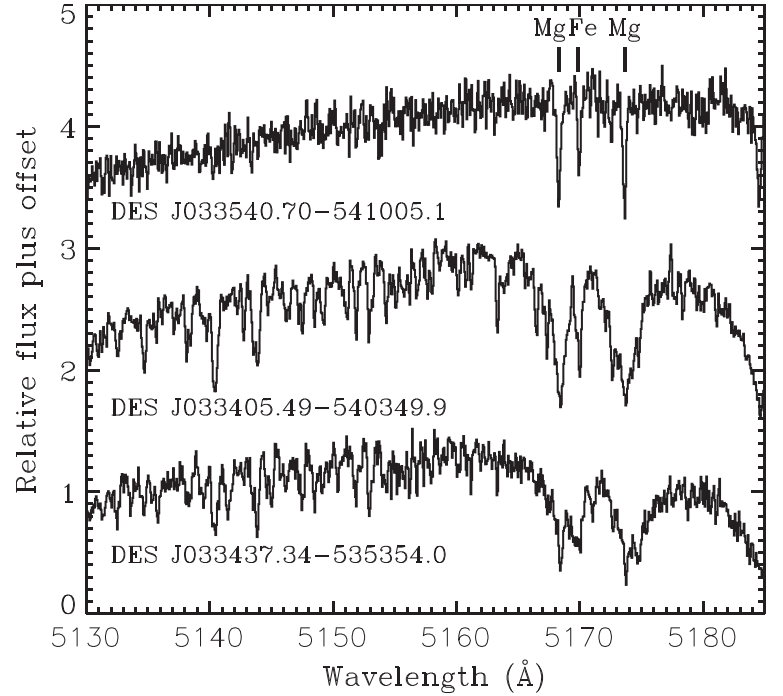

Figure 2. Magellan/M2FS spectra in the $\mathrm{Mg} b$ triplet region for three stars near the edge of the Ret II velocity distribution. The wavelengths of two $\mathrm{Mg}$ lines and an Fe line are marked in the bottom panel, and the third component of the $\mathrm{Mg}$ triplet is just visible at a wavelength of $5185 \AA$ at the right edge of each spectrum. The spectrum of DES J033540.70-541005.1 (top) appears similar to that of a Ret II member, but the color, spatial position, and velocity offset of this star make that classification unlikely. The very strong $\mathrm{Mg}$ absorption in DES J033405.49-540349.9 (middle), as well as the wealth of other absorption features on the blue side of the spectrum, indicate that the star is more metal-rich than would be expected for a system as small as Ret II DES J033437.34-535354.0 (bottom) is a double-lined binary star with a velocity separation of $\sim 60 \mathrm{~km} \mathrm{~s}^{-1}$. The redshifted absorption component from the secondary star is most visible in the middle line of the $\mathrm{Mg}$ triplet.

isochrone. Since it is near the base of the giant branch, the photometric uncertainties could contribute to this offset in color, and we consider DES J033544.18-540150.0 a likely member of Ret II.

Because the stars for which membership is plausible have velocities quite similar to that of Ret II (and in some cases have large uncertainties), including or excluding them from the member sample does not have any significant effect on the properties we derive for Ret II in Section 4. We show the

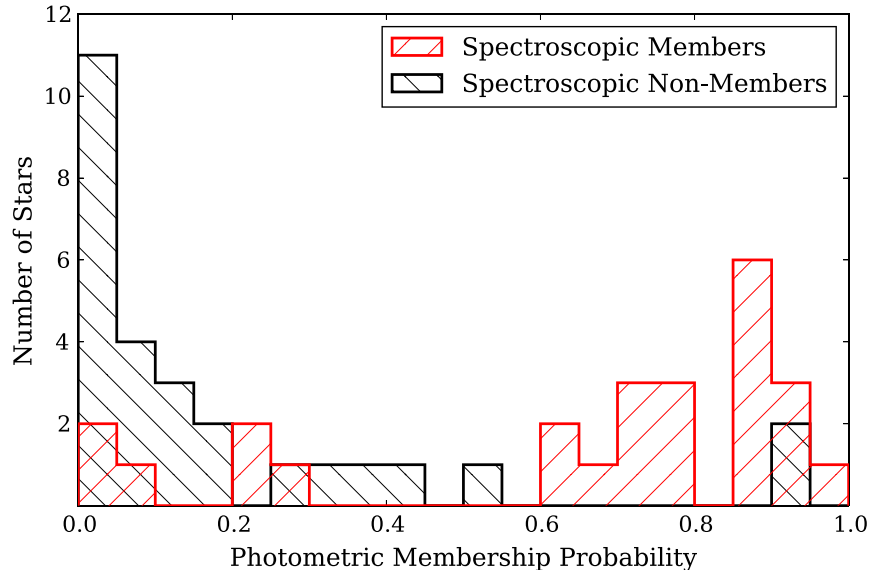

Figure 3. Comparison of photometric membership probabilities determined from a maximum-likelihood fit to the DES data and spectroscopic membership as determined from the velocity measured by M2FS.

correspondence between M2FS spectroscopic members and photometric membership probability in Figure 3.

\subsubsection{GIRAFFE and GMOS}

We also identify a handful of Ret II members in the GIRAFFE and GMOS data sets that were not observed with M2FS. We use a velocity measurement based on the Paschen lines to confirm that the candidate blue HB (BHB) star DES J033539.85-540458.1 (Section 3.4) observed by GMOS is indeed a member of Ret II, with a velocity of $69 \pm 6 \mathrm{~km} \mathrm{~s}^{-1}$. The GIRAFFE targets included a bright $(g \sim 16.5)$ star at $\left(\alpha_{2000}, \delta_{2000}\right)=(03: 35: 23.85$, $-54: 04: 07.5)$ that was omitted from our photometric catalog and M2FS observations because it is saturated in the coadded DES images. However, the spectrum of the star makes clear that it is very metal-poor and is within a few $\mathrm{km} \mathrm{s}^{-1}$ of the systemic velocity of Ret II. While the magnitudes derived from individual DES frames place it slightly redder than the isochrone that best matches the lower red giant branch of Ret II, it is also located inside the half-light radius, and is very likely a member. In fact, it is probably the brightest star in any of the ultra-faint dwarfs. 
Another GIRAFFE target, DES J033548.04-540349.8, was not included in the M2FS sample because it was assigned a photometric membership probability of zero by the maximumlikelihood analysis (Bechtol et al. 2015). It is very slightly redder (relative to the isochrone) than the bulk of the Ret II stars, but it is located within the half-light radius and has a velocity $\sim 1 \mathrm{~km} \mathrm{~s}^{-1}$ from the systemic velocity. The metallicity we determine from the GIRAFFE spectrum is low, but somewhat more metal-rich than any of the other member stars, perhaps consistent with its color. The Mg I $\lambda 8807$ line advocated by Battaglia \& Starkenburg (2012) as a discriminant between dwarf-galaxy red giants and foreground main sequence stars is weak (EW $=0.1 \AA$ ), suggesting that it is a member of Ret II, but since it would be the highest metallicity star in the galaxy we regard this conclusion as tentative. Although all three of these stars are likely members of Ret II, we do not include them in the determination of the kinematic properties in Section 4.1 because they have not been placed accurately enough in the same velocity reference frame.

We classify the GIRAFFE target DES J033524.96-540230.7 as a non-member based on its extremely broad CaT lines, despite its overlap with the Ret II velocity distribution.

Finally, we note that for DES J033515.17-540843.0, the M2FS and GIRAFFE velocity measurements differ by $8.2 \pm 3.5 \mathrm{~km} \mathrm{~s}^{-1}$. The significance of this difference is $2.4 \sigma$, corresponding to a $p$-value of $2 \%$. While we cannot rule out the possibility that this difference in velocity is attributable to chance or systematics, we suggest that this star may be a binary system in Ret II. Orbital motion of $8 \mathrm{~km} \mathrm{~s}^{-1}$ over a time span of 18 days is consistent with the handful of previously detected binaries in ultra-faint dwarf galaxies (Koposov et al. 2011; Koch et al. 2014).

\subsubsection{Foreground Modeling}

Finally, we consider the expected contamination from foreground stars in a statistical sense. We use the Besançon (Robin \& Reylé 2003) and TRILEGAL (Girardi et al. 2005; Vanhollebeke et al. 2009) Galactic stellar models to calculate the expected velocity distribution of foreground stars in the region of Ret II. We select stars within $0.02 \mathrm{mag}$ of the composite isochrone (Section 2.1) and with $17<g<20.6$. From the resulting velocity distribution, we find that $\sim 10 \%$ of foreground stars passing these photometric cuts have a velocity consistent with the heliocentric velocity peak of Ret II $\left(55-70 \mathrm{~km} \mathrm{~s}^{-1}\right)$. We set the overall normalization for the number of foreground stars by assuming that all stars in the sideband of the peak $\left(v<40\right.$ or $\left.v>90 \mathrm{~km} \mathrm{~s}^{-1}\right)$ belong to the foreground distribution. The expected number of foreground stars within the peak of ranges from 3.0 (Besançon) to 4.1 (TRILEGAL). Given the small number statistics, this is consistent with the results of the membership analysis above. Under these assumptions, the foreground-only hypothesis for the Ret II velocity peak is excluded at $p \approx 10^{-12}$.

\subsection{HB Stars}

The DES photometric catalog contains three candidate BHB stars, three candidate red HB stars, and one star located slightly below the $\mathrm{HB}$ at an intermediate color that could be consistent with the RR Lyrae instability strip. We targeted all seven of these stars with M2FS, but were only able to determine velocities for five of them. For the BHB stars, we confirm DES J033618.68-535745.1 as a member of Ret II, but we do not detect any spectral features for DES J033539.85-540458.1, and DES J033612.7-535602.2 was observed with a fiber with $3 \%$ throughput so the spectrum was not useful. As mentioned in Section 3.3, our GMOS spectrum of DES J033539.85-540458.1 confirms it as a Ret II member. The velocities of the three RHB candidates indicate that they are not members. The final HB candidate, DES J033341.71-540007.3, has a measured velocity of $34.4 \pm 1.2 \mathrm{~km} \mathrm{~s}^{-1}$, which could be consistent with membership in Ret II if it were an RR Lyrae variable, but we do not detect photometric variations for it or any of the BHB stars in the 2-4 DES single-epoch images per filter available in this field.

\section{DISCUSSION}

\subsection{Velocity Dispersion and Mass}

After identifying likely members of Ret II with a combination of photometry, velocity, and metallicity information, we can calculate the systemic velocity and velocity dispersion of Ret II. For the sample of 25 member stars with M2FS velocity measurements identified in Section 3, we find a systemic velocity of $v_{\text {hel }}=62.8 \pm 0.5 \mathrm{~km} \mathrm{~s}^{-1}$. Using the method of Walker et al. (2006), we determine a maximum-likelihood estimate for the velocity dispersion of $\sigma_{v}=3.3 \pm 0.7 \mathrm{~km} \mathrm{~s}^{-1}$. If we instead rely on the GIRAFFE data set (which includes 17 member stars), we obtain fully compatible values of $v_{\text {hel }}=63.3 \pm 0.8 \mathrm{~km} \mathrm{~s}^{-1}$ and $\sigma_{v}=3.5 \pm 1.0 \mathrm{~km} \mathrm{~s}^{-1}$. The velocity dispersion of Ret II is also robust to membership uncertainties; decisions about including or excluding the plausible member stars discussed in Section 3.3 change the calculated dispersion by less than the stated uncertainty. Only by adding stars that are very unlikely to be members for multiple reasons can the best-fit velocity dispersion be significantly increased. In principle, the low velocity dispersion of Ret II increases the likelihood that the measured dispersion could be artificially inflated because of the orbital motions of binary stars. While our observations do not span a long enough time baseline to have much sensitivity to binaries, previous studies have shown that even in the smallest dwarfs binary stars do not significantly inflate the observed velocity dispersion (Martinez et al. 2011; Simon et al. 2011).

Wolf et al. (2010) showed that, independent of the velocity anisotropy of a stellar system, the mass enclosed within the half-light radius can be accurately computed as

$$
M_{1 / 2}=930\left(\frac{\sigma_{v}^{2}}{\mathrm{~km}^{2} \mathrm{~s}^{-2}}\right)\left(\frac{R_{1 / 2}}{\mathrm{pc}}\right) M_{\odot} .
$$

Using this relation, the mass within the elliptical half-light radius of Ret II $\left(R_{1 / 2}=55 \pm 5 \mathrm{pc}\right.$; Bechtol et al. $2015)$ is $5.6 \pm 2.4 \times 10^{5} M_{\odot}$. The absolute magnitude of $M_{V}=-3.6 \pm 0.1$ from Bechtol et al. (2015) translates to a luminosity of $2360 L_{\odot}$, leading to a mass-to-light ratio of $470 \pm 210 M_{\odot} / L_{\odot}$. This value is consistent with the inverse correlation between mass-to-light ratio and luminosity for other Local Group dwarf galaxies, although Ret II is on the low end for galaxies of similar luminosity (see Figure 4). 

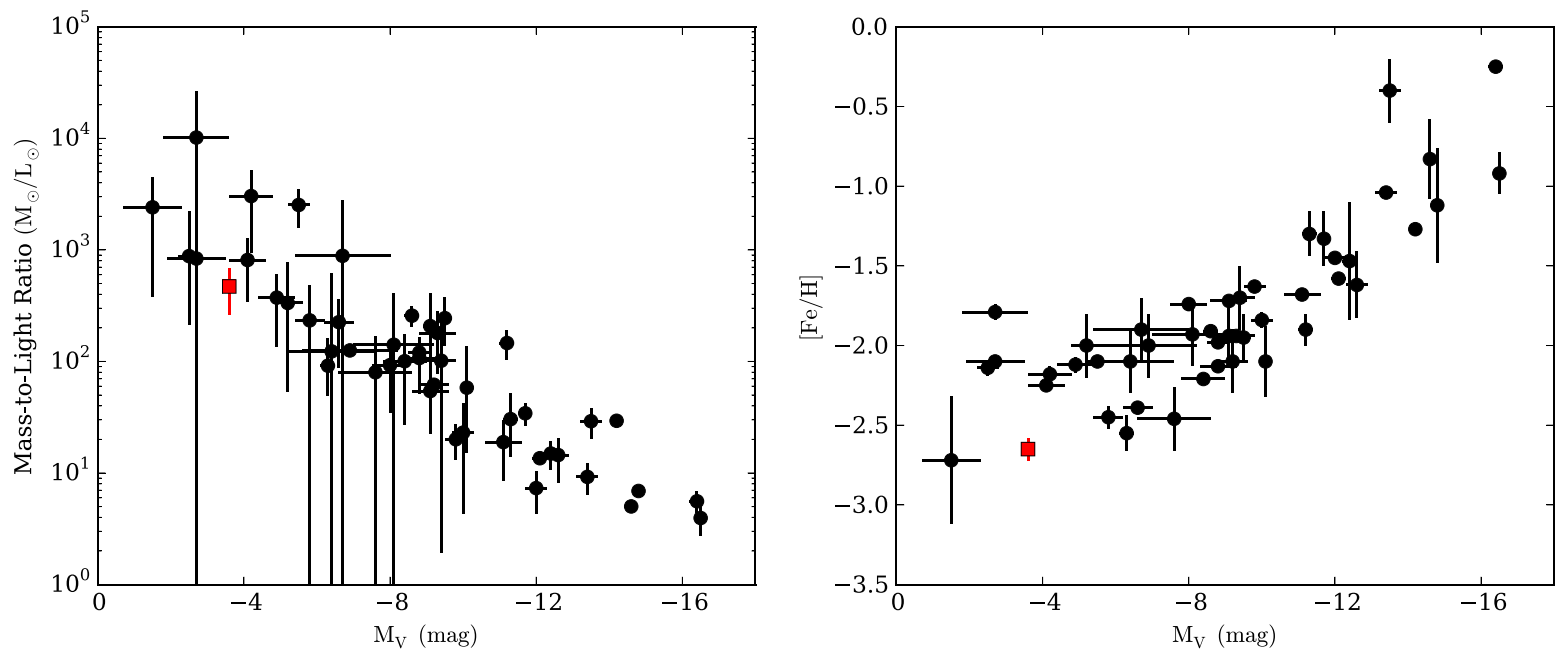

Figure 4. Mass-to-light ratio (left) and metallicity (right) of Ret II compared to other galaxies within 1Mpc. Mass-to-light ratios were calculated from the velocity dispersions, half-light radii, and absolute magnitudes compiled by McConnachie (2012) in order to maintain consistency with the value we report for Ret II. Metallicities were also adopted from the McConnachie (2012) compilation, with updates from Kirby et al. (2013) where newer measurements were available.

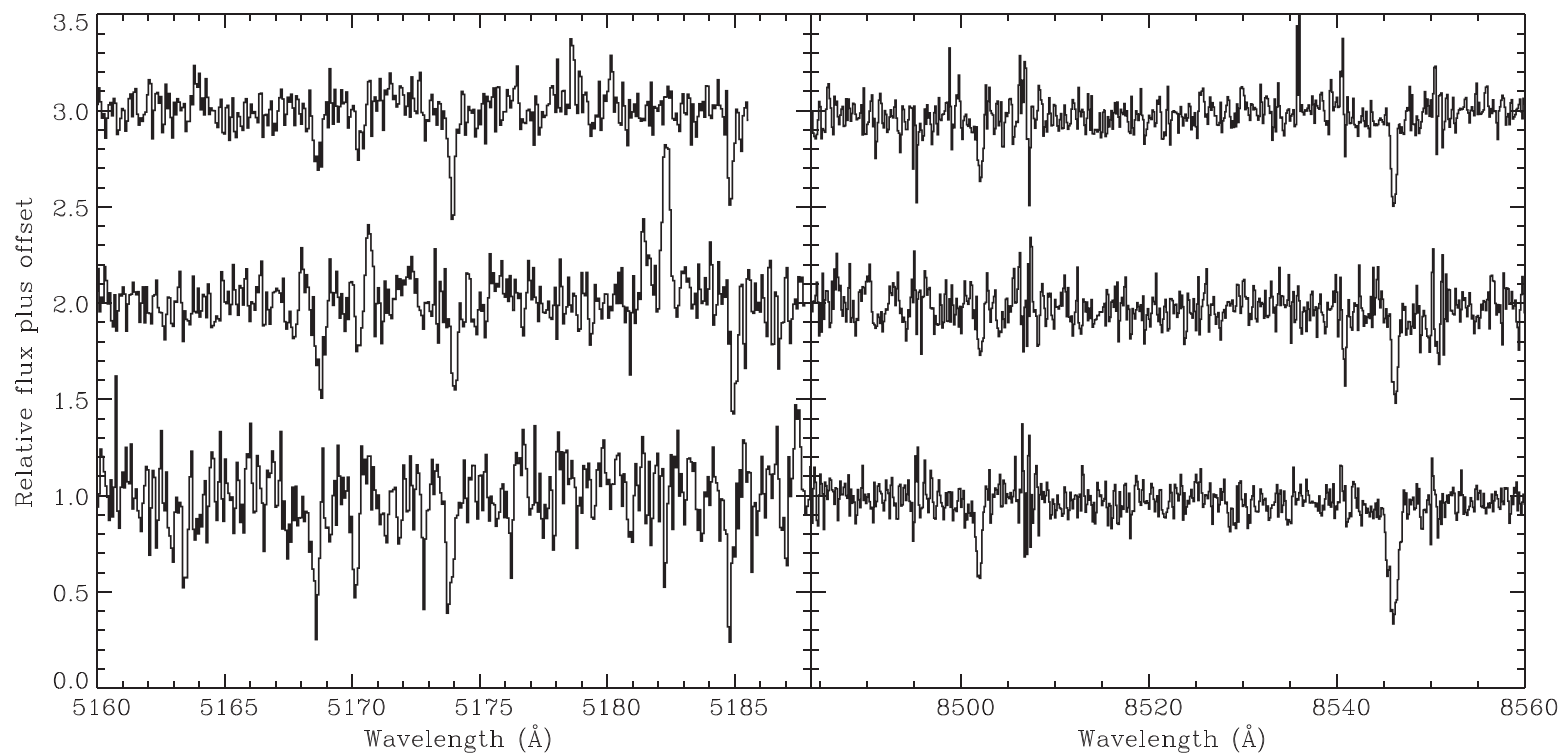

Figure 5. (Left) Magellan/M2FS spectra in the $\mathrm{Mg} b$ triplet region for three Ret II member stars covering a range of line strengths. From top to bottom, the stars are DES J033556.28-540316.3, DES J033454.24-540558.0, and DES J033457.57-540531.4. These stars span only $0.1 \mathrm{mag}$ in luminosity and 0.08 mag in $g-r$ color, so their effective temperatures and surface gravities should be very similar. Any differences in line strength therefore translate directly into chemical abundance differences. The apparent emission features near $5182 \AA$ in the spectrum of DES J033454.24-540558.0 are contamination by the Littrow ghost (Burgh et al. 2007). (Right) VLT/GIRAFFE spectra of the bluer two CaT lines for the same stars.

\subsection{Metallicity Spread}

Even a visual inspection of the M2FS spectra reveals clear chemical abundance differences within the Ret II member sample. In Figure 5 we display spectra of three of the stars within the clump of Ret II members at $g \approx 18.9$ in Figure $1(\mathrm{a})$. The absolute strength of the $\mathrm{Mg}$ absorption and the $\mathrm{Fe}$ absorption, as well as the ratio between the two, varies from star to star, demonstrating that Ret II is not chemically homogeneous.

The CaT metallicity measurements from the VLT/GIRAFFE spectra provide a more quantitative demonstration of the spread in overall metallicity from star to star in Ret II. We find member stars spanning more than $1 \mathrm{dex}$ in iron abundance, ranging from $[\mathrm{Fe} / \mathrm{H}]=-2.0$ to $[\mathrm{Fe} / \mathrm{H}]=-3.3$. The individual metallicities are displayed as a function of radius in
Figure 6(b). There is no indication of a metallicity gradient within Ret II. The metallicity spread, calculated with the same technique we used in Section 4.1 for the velocity dispersion, is $0.28 \pm 0.09 \mathrm{dex}$. If we instead employ the definition of Kirby et al. (2011), we find a similar spread. Both this dispersion and the presence of several extremely metal-poor stars at $[\mathrm{Fe} / \mathrm{H}]<-3$ demonstrate that Ret II has the chemical characteristics of a dwarf galaxy rather than a globular cluster. The mean metallicity of Ret II is $[\mathrm{Fe} / \mathrm{H}]=-2.65 \pm 0.07$, ranking it along with Segue 1 as the most metal-poor galaxies known. This metallicity measurement places Ret II somewhat below the stellar mass-metallicity relationship determined by Kirby et al. (2013) and argues against the existence of a metallicity floor for dwarf galaxies (Figure 4). The kinematic and chemical properties of Ret II are summarized in Table 2. 

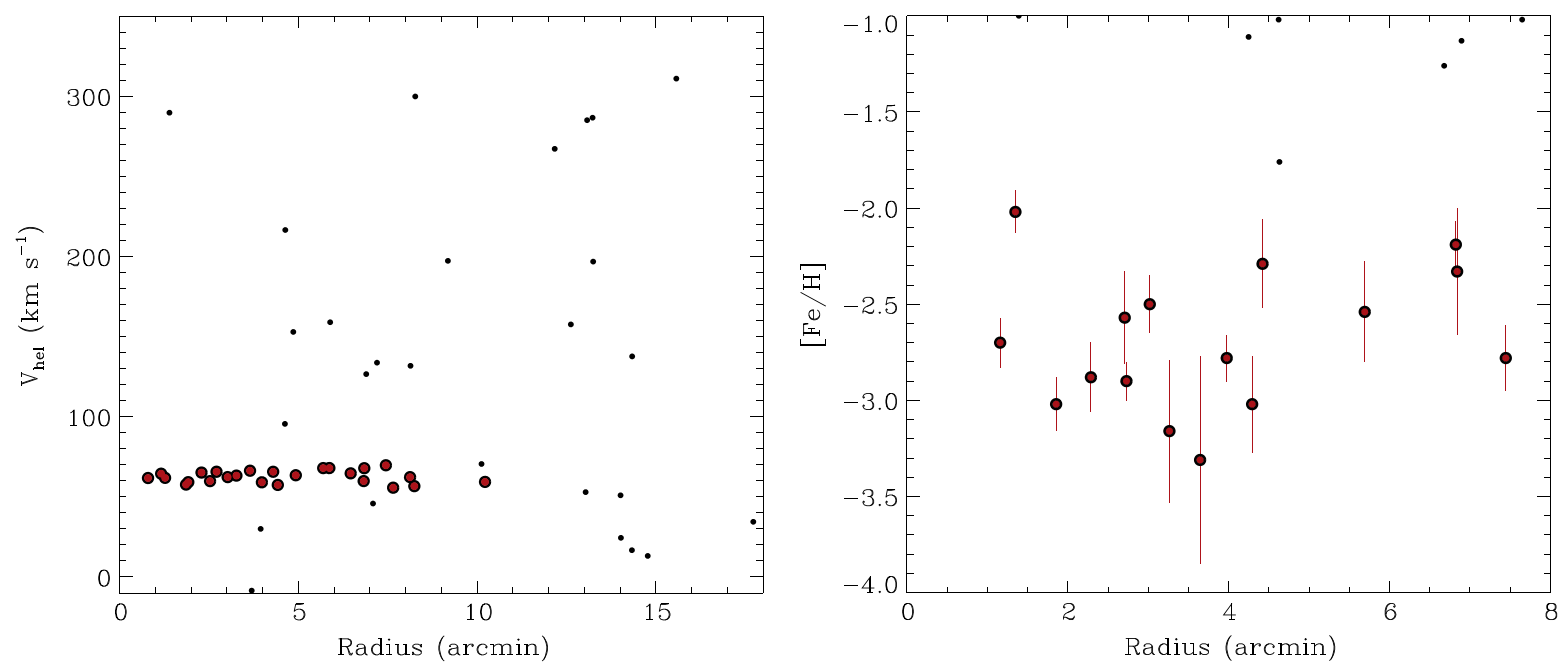

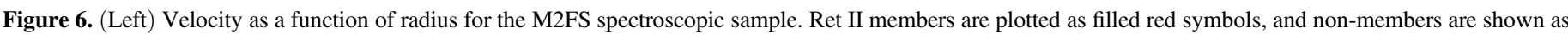

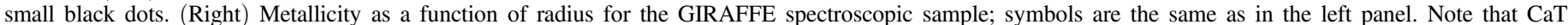

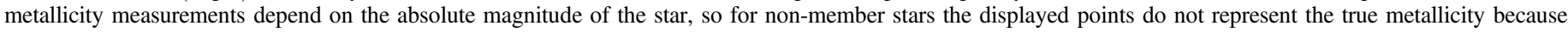
those stars are not at the distance of Ret II.

\subsection{Reticulum II and the Magellanic Clouds}

The proximity of the DES satellites to the Magellanic Clouds suggests that some or all of them may have originated in a Magellanic group. Ret II is the closest of these objects to the Magellanic Clouds, with a three-dimensional separation of just $23 \pm 3 \mathrm{kpc}$. The radial velocity of Ret II differs from that of the LMC by $199 \mathrm{~km} \mathrm{~s}^{-1}$, which represents a lower limit on the difference in their space motions. The total mass of the LMC out to a distance of $23 \mathrm{kpc}$ is not known, but Kallivayalil et al. (2013) argue that reasonable bounds are $3 \times 10^{10} M_{\odot}$ to $2.5 \times 10^{11} M_{\odot}$. Using DES imaging, Balbinot et al. (2015) determine a truncation radius of $13.5 \pm 0.08 \mathrm{kpc}$ for the LMC. If this truncation is tidal in origin, the favored mass is at the lower limit of the range suggested by Kallivayalil et al. (2013). For a mass of $3 \times 10^{10} M_{\odot}$, the escape velocity of the LMC at $d=23 \mathrm{kpc}$ is only $106 \mathrm{~km} \mathrm{~s}^{-1}$, and for $M_{\mathrm{LMC}}<10^{11} M_{\odot}$ the escape velocity is less than $200 \mathrm{~km} \mathrm{~s}^{-1}$. Only if the LMC mass is higher than this value and the tangential velocity of Ret II is similar to that of the LMC could Ret II currently be a bound satellite of the Magellanic Clouds. Of course, even if Ret II is not bound to the LMC now, it is possible that it was an LMC satellite at earlier times and was stripped from that host by the tidal field of the Milky Way more recently. A proper motion measurement of Ret II will be needed to conclusively determine its origin, and its location just $32 \mathrm{kpc}$ away from the Sun should enable the proper motion to be determined in the next few years.

The observed velocity distribution of high velocity $\left(v_{\text {hel }}>100 \mathrm{~km} \mathrm{~s}^{-1}\right)$ stars displayed in Figure 1(c) appears inconsistent with the distribution of velocities for Milky Way stars predicted by Galactic models (Section 3.3.3). In particular, there are unexpected concentrations of stars around $145 \mathrm{~km} \mathrm{~s}^{-1}$ (six stars) and $300 \mathrm{~km} \mathrm{~s}^{-1}$ (five stars). The former group of stars is near the heliocentric velocity of the SMC (145.6 $\mathrm{km} \mathrm{s}^{-1}$; Harris \& Zaritsky 2006), suggesting that these might be distant SMC stars. If they are at the distance of the SMC, they are at projected separations of $27 \mathrm{kpc}$, approximately twice as far out as the most distant currently known SMC population (Nidever et al. 2011), and have therefore likely been tidally stripped. The higher velocity stars have very similar velocities to the Magellanic Stream gas a few degrees away from Ret II, and could represent a stellar counterpart of the Stream.

\subsection{J-factor}

It is posited that dark matter particles could self-annihilate to produce gamma-rays (e.g., Gunn et al. 1978; Bergström \& Snellman 1988; Baltz et al. 2008). The large dark matter content, relative proximity, and low astrophysical foregrounds of dwarf galaxies make them promising targets for the detection of these gamma-rays. The predicted signal from the annihilation of dark matter particles is proportional to the lineof-sight integral through the square of the dark matter density (e.g., Baltz et al. 2008),

$$
J(\Delta \Omega)=\int_{\Delta \Omega} \int_{\text {l.o.s. }} \rho_{\mathrm{DM}}^{2}(\boldsymbol{r}) d s d \Omega^{\prime} .
$$

Here, $\rho_{\mathrm{DM}}(\boldsymbol{r})$ is the dark matter particle density, and the integral is performed over a solid angle $\Delta \Omega$. The $J$-factor is derived by modeling the velocities using the spherical Jeans equation, with assumptions on the theoretical priors for the parameters that describe the dark matter halo (e.g., Strigari et al. 2008; Essig et al. 2009; Charbonnier et al. 2011; Martinez 2015; Geringer-Sameth et al. 2015a). Here, we model the dark matter halo as a generalized Navarro-Frenk-White profile (Navarro et al. 1997). We use flat, "uninformative" priors on the dark matter halo parameters (see Essig et al. 2009) and assume a constant stellar velocity anisotropy. Using this procedure, we find an integrated $J$-factor for Ret II of $\log _{10}(J)=18.8 \pm 0.6 \mathrm{GeV}^{2} \mathrm{~cm}^{-5}$ within an angular cone of radius $0: 2$, and $\log _{10}(J)=18.9 \pm 0.6 \mathrm{GeV}^{2} \mathrm{~cm}^{-5}$ within $0: 5$. This latter value assumes that the dark matter halo extends beyond the radius of the outermost spectroscopically confirmed star, but truncates within the estimated tidal radius for the dark matter halo $(\sim 1 \mathrm{kpc})$. The quoted uncertainties are $1 \sigma$, and are estimated by modeling the posterior probability density function of $\log _{10}(J)$ as a Gaussian. Note that the uncertainty 
Table 1

Velocity and Metallicity Measurements

\begin{tabular}{|c|c|c|c|c|c|c|c|c|c|c|}
\hline ID & $\begin{array}{l}\text { R.A. } \\
\text { (deg) }\end{array}$ & $\begin{array}{l}\text { Decl. } \\
\text { (deg) }\end{array}$ & $\begin{array}{c}g^{\mathrm{a}} \\
(\mathrm{mag})\end{array}$ & $\begin{array}{c}r^{\mathrm{a}} \\
(\mathrm{mag})\end{array}$ & $\begin{array}{c}v \\
\left(\mathrm{~km} \mathrm{~s}^{-1}\right)\end{array}$ & $\mathrm{EW}_{\text {GIRAFFE }}$ & {$[\mathrm{Fe} / \mathrm{H}]_{\mathrm{GIRAFFE}}$} & $\mathrm{EW}_{\mathrm{GMOS}}$ & {$[\mathrm{Fe} / \mathrm{H}]_{\mathrm{GMOS}}$} & MEM \\
\hline & & & & & M2FS & & & & & \\
\hline DES J033341.71-540007.3 & 53.42377 & -54.00201 & 18.26 & 18.20 & $34.42 \pm 1.16$ & $\ldots$ & $\ldots$ & $\ldots$ & $\ldots$ & NM \\
\hline DES J033405.49-540349.9 & 53.52287 & -54.06387 & 17.58 & 16.99 & $50.96 \pm 0.94$ & $\cdots$ & $\cdots$ & $\cdots$ & $\cdots$ & NM \\
\hline DES J033413.06-535956.1 & 53.55441 & -53.99892 & 19.45 & 18.99 & $196.81 \pm 1.44$ & $\ldots$ & $\ldots$ & $\ldots$ & $\ldots$ & NM \\
\hline DES J033413.94-540934.4 & 53.55807 & -54.15956 & 18.26 & 17.68 & $16.70 \pm 1.06$ & $\ldots$ & $\ldots$ & $\ldots$ & $\ldots$ & NM \\
\hline DES J033418.32-541006.2 & 53.57632 & -54.16839 & 18.02 & 17.45 & $24.41 \pm 0.98$ & $\ldots$ & $\ldots$ & $\ldots$ & $\ldots$ & NM \\
\hline DES J033429.94-5411111.8 & 53.62474 & -54.18661 & 19.77 & 19.32 & $286.61 \pm 1.55$ & $\ldots$ & $\cdots$ & $\ldots$ & $\ldots$ & NM \\
\hline DES J033430.07-540922.2 & 53.62528 & -54.15618 & 18.17 & 17.85 & $267.19 \pm 0.97$ & $\ldots$ & $\ldots$ & $\ldots$ & $\ldots$ & NM \\
\hline DES J033436.70-540645.0 & 53.65291 & -54.11249 & 18.85 & 18.29 & $70.52 \pm 1.01$ & $\cdots$ & $\cdots$ & $\cdots$ & $\cdots$ & NM? \\
\hline DES J033437.34-535354.0 & 53.65557 & -53.89832 & 17.69 & 17.11 & $52.94 \pm 1.10$ & $\ldots$ & $\ldots$ & $\ldots$ & $\ldots$ & NM \\
\hline DES J033437.98-541359.7 & 53.65825 & -54.23324 & 20.55 & 20.07 & $137.64 \pm 2.58$ & $\cdots$ & $\cdots$ & $\cdots$ & $\cdots$ & NM \\
\hline DES J033439.66-540754.4 & 53.66523 & -54.13177 & 19.38 & 18.87 & $59.32 \pm 1.84$ & $\ldots$ & $\ldots$ & $\ldots$ & $\ldots$ & $\mathrm{M}$ \\
\hline DES J033439.81-540058.5 & 53.66587 & -54.01626 & 18.21 & 17.93 & $197.28 \pm 1.00$ & $\ldots$ & $\ldots$ & $\ldots$ & $\ldots$ & NM \\
\hline DES J033447.94-540525.0 & 53.69974 & -54.09028 & 17.52 & 16.92 & $62.28 \pm 1.03$ & $\ldots$ & $\ldots$ & $\ldots$ & $\ldots$ & M \\
\hline DES J033449.20-535019.7 & 53.70502 & -53.83881 & 17.62 & 17.02 & $13.13 \pm 0.93$ & $\cdots$ & $\cdots$ & $\cdots$ & $\cdots$ & NM \\
\hline DES J033453.23-541403.9 & 53.72180 & -54.23443 & 20.59 & 20.19 & $285.07 \pm 2.73$ & $\cdots$ & $\cdots$ & $\cdots$ & $\cdots$ & NM \\
\hline DES J033453.50-540454.6 & 53.72291 & -54.08182 & 20.18 & 19.80 & $133.74 \pm 2.58$ & $\ldots$ & $\ldots$ & $\cdots$ & $\cdots$ & NM \\
\hline DES J033454.24-540558.0 & 53.72600 & -54.09945 & 18.95 & 18.44 & $69.70 \pm 1.38$ & $1.69 \pm 0.25$ & $-2.78 \pm 0.17$ & $\ldots$ & $\ldots$ & $\mathrm{M}$ \\
\hline DES J033457.57-540531.4 & 53.73988 & -54.09206 & 18.94 & 18.42 & $59.84 \pm 1.17$ & $2.77 \pm 0.23$ & $-2.19 \pm 0.12$ & $\ldots$ & $\ldots$ & M \\
\hline DES J033502.50-540354.3 & 53.76041 & -54.06507 & 19.24 & 18.75 & $67.94 \pm 1.14$ & $1.98 \pm 0.43$ & $-2.54 \pm 0.26$ & $\ldots$ & $\ldots$ & M \\
\hline DES J033502.87-540109.8 & 53.76196 & -54.01940 & 20.36 & 19.93 & $67.96 \pm 3.48$ & $\ldots$ & $\ldots$ & $\cdots$ & $\cdots$ & M \\
\hline DES J033506.56-540604.3 & 53.77734 & -54.10120 & 19.79 & 19.28 & $158.97 \pm 1.36$ & $\ldots$ & $\cdots$ & $\cdots$ & $\cdots$ & NM \\
\hline DES J033509.50-540229.7 & 53.78959 & -54.04158 & 18.24 & 17.76 & $95.55 \pm 0.96$ & $5.72 \pm 0.29$ & $\ldots$ & $\cdots$ & $\cdots$ & NM \\
\hline DES J033511.66-540321.8 & 53.79858 & -54.05606 & 19.31 & 18.83 & $65.64 \pm 1.34$ & $1.27 \pm 0.27$ & $-3.02 \pm 0.25$ & $\cdots$ & $\cdots$ & M \\
\hline DES J033513.73-540456.7 & 53.80722 & -54.08242 & 19.70 & 19.24 & $57.43 \pm 2.38$ & $2.26 \pm 0.40$ & $-2.29 \pm 0.23$ & $\cdots$ & $\cdots$ & M \\
\hline DES J033514.01-540558.2 & 53.80839 & -54.09949 & 20.01 & 19.57 & $63.47 \pm 1.45$ & $\ldots$ & $\ldots$ & $\cdots$ & $\cdots$ & M \\
\hline DES J033515.17-540843.0 & 53.81322 & -54.14529 & 19.73 & 19.25 & $67.87 \pm 1.36$ & $2.19 \pm 0.58$ & $-2.33 \pm 0.33$ & $\ldots$ & $\ldots$ & M \\
\hline DES J033517.01-540403.0 & 53.82088 & -54.06751 & 19.72 & 19.24 & $66.31 \pm 1.40$ & $0.93 \pm 0.38$ & $-3.31 \pm 0.54$ & $1.37 \pm 0.20$ & $-2.86 \pm 0.18$ & $\mathrm{M}$ \\
\hline DES J033520.37-541816.9 & 53.83486 & -54.30470 & 20.53 & 20.18 & $311.02 \pm 2.83$ & $\ldots$ & $\ldots$ & $\ldots$ & $\ldots$ & NM \\
\hline DES J033520.97-540348.2 & 53.83736 & -54.06338 & 18.95 & 18.46 & $62.31 \pm 1.06$ & $2.16 \pm 0.26$ & $-2.50 \pm 0.15$ & $1.15 \pm 0.28$ & $-3.20 \pm 0.30$ & $\mathrm{M}$ \\
\hline DES J033524.00-540226.7 & 53.85002 & -54.04075 & 20.31 & 19.89 & $59.81 \pm 1.83$ & $\ldots$ & $\ldots$ & $\ldots$ & $\ldots$ & M \\
\hline DES J033531.14-540148.2 & 53.87975 & -54.03007 & 17.64 & 17.07 & $57.66 \pm 0.96$ & $1.72 \pm 0.20$ & $-3.02 \pm 0.14$ & $1.88 \pm 0.26$ & $-2.92 \pm 0.16$ & M \\
\hline DES J033533.71-535025.4 & 53.89045 & -53.84038 & 18.75 & 18.25 & $157.61 \pm 2.22$ & $\ldots$ & $\ldots$ & $\ldots$ & $\ldots$ & NM \\
\hline DES J033535.44-540254.9 & 53.89766 & -54.04857 & 20.68 & 20.35 & $61.74 \pm 4.79$ & $\ldots$ & $\ldots$ & $\ldots$ & $\ldots$ & M \\
\hline DES J033536.94-535445.1 & 53.90391 & -53.91253 & 20.56 & 20.16 & $299.87 \pm 2.00$ & $\ldots$ & $\ldots$ & $\ldots$ & $\ldots$ & NM \\
\hline DES J033537.06-540401.2 & 53.90442 & -54.06701 & 18.57 & 18.03 & $64.44 \pm 1.14$ & $1.94 \pm 0.21$ & $-2.70 \pm 0.13$ & $1.67 \pm 0.48$ & $-2.86 \pm 0.32$ & M \\
\hline DES J033540.70-541005.1 & 53.91957 & -54.16809 & 18.23 & 17.92 & $45.83 \pm 0.99$ & $\ldots$ & $\ldots$ & $\ldots$ & $\ldots$ & NM \\
\hline DES J033544.18-540150.0 & 53.93409 & -54.03056 & 20.40 & 19.91 & $61.86 \pm 1.99$ & $\ldots$ & $\ldots$ & $\ldots$ & $\ldots$ & M? \\
\hline DES J033546.17-540733.9 & 53.94235 & -54.12608 & 19.23 & 18.74 & $216.54 \pm 1.16$ & $3.51 \pm 0.41$ & $\ldots$ & $\ldots$ & $\ldots$ & NM \\
\hline DES J033547.47-535926.5 & 53.94781 & -53.99068 & 21.25 & 20.99 & $-8.53 \pm 4.78$ & $\ldots$ & $\cdots$ & $\ldots$ & $\cdots$ & NM \\
\hline DES J033549.96-540321.5 & 53.95816 & -54.05596 & 19.66 & 19.21 & $289.73 \pm 1.23$ & $4.93 \pm 0.36$ & $\cdots$ & $4.65 \pm 0.59$ & $\cdots$ & NM \\
\hline DES J033550.10-540139.2 & 53.95873 & -54.02756 & 19.69 & 19.26 & $59.15 \pm 8.25$ & $\ldots$ & $\ldots$ & $\ldots$ & $\ldots$ & $\mathrm{M}$ ? \\
\hline DES J033552.08-540733.9 & 53.96700 & -54.12608 & 20.56 & 20.21 & $152.93 \pm 2.52$ & $\ldots$ & $\ldots$ & $\ldots$ & $\ldots$ & NM \\
\hline DES J033556.28-540316.3 & 53.98449 & -54.05452 & 18.85 & 18.37 & $65.15 \pm 1.18$ & $1.56 \pm 0.24$ & $-2.88 \pm 0.18$ & $1.24 \pm 0.28$ & $-3.14 \pm 0.27$ & M \\
\hline DES J033558.15-540204.8 & 53.99228 & -54.03466 & 19.30 & 18.82 & $65.67 \pm 1.12$ & $1.92 \pm 0.38$ & $-2.57 \pm 0.24$ & $1.33 \pm 0.48$ & $-2.97 \pm 0.40$ & M \\
\hline DES J033601.76-540405.5 & 54.00734 & -54.06819 & 19.56 & 19.06 & $63.25 \pm 1.40$ & $1.08 \pm 0.32$ & $-3.16 \pm 0.37$ & $\ldots$ & $\ldots$ & M \\
\hline DES J033606.25-540144.5 & 54.02604 & -54.02903 & 20.39 & 19.93 & $29.99 \pm 4.19$ & $\ldots$ & $\ldots$ & $\ldots$ & $\ldots$ & NM \\
\hline
\end{tabular}


Table 1

(Continued)

\begin{tabular}{|c|c|c|c|c|c|c|c|c|c|c|}
\hline ID & $\begin{array}{l}\text { R.A. } \\
\text { (deg) }\end{array}$ & $\begin{array}{l}\text { Decl. } \\
\text { (deg) }\end{array}$ & $\begin{array}{c}g^{\mathrm{a}} \\
(\mathrm{mag})\end{array}$ & $\begin{array}{c}r^{\mathrm{a}} \\
(\mathrm{mag})\end{array}$ & $\begin{array}{c}v \\
\left(\mathrm{~km} \mathrm{~s}^{-1}\right)\end{array}$ & $\mathrm{EW}_{\text {GIRAFFE }}$ & {$[\mathrm{Fe} / \mathrm{H}]_{\text {GIRAFFE }}$} & $\mathrm{EW}_{\mathrm{GMOS}}$ & {$[\mathrm{Fe} / \mathrm{H}]_{\mathrm{GMOS}}$} & MEM \\
\hline DES J033607.75-540235.6 & 54.03230 & -54.04321 & 17.43 & 16.82 & $59.07 \pm 0.92$ & $2.22 \pm 0.21$ & $-2.78 \pm 0.12$ & $\ldots$ & $\ldots$ & M \\
\hline DES J033618.68-535745.1 & 54.07784 & -53.96254 & 18.04 & 18.20 & $55.71 \pm 3.23$ & $\ldots$ & $\ldots$ & $\ldots$ & $\ldots$ & M \\
\hline DES J033621.86-540040.7 & 54.09109 & -54.01130 & 20.30 & 19.87 & $64.66 \pm 1.75$ & $\ldots$ & $\ldots$ & $\ldots$ & $\ldots$ & M \\
\hline DES J033622.83-535955.5 & 54.09513 & -53.99876 & 18.58 & 18.12 & $126.61 \pm 0.94$ & $5.25 \pm 0.33$ & $\ldots$ & $\ldots$ & $\ldots$ & NM \\
\hline DES J033635.75-540156.9 & 54.14895 & -54.03248 & 18.05 & 17.61 & $131.78 \pm 0.94$ & $\ldots$ & $\ldots$ & $\ldots$ & $\ldots$ & NM \\
\hline DES J033635.78-540120.2 & 54.14909 & -54.02228 & 20.17 & 19.73 & $56.68 \pm 1.91$ & $\cdots$ & $\cdots$ & $\cdots$ & $\cdots$ & M? \\
\hline \multicolumn{11}{|c|}{ GIRAFFE } \\
\hline DES J033523.85-540407.5 & 53.84938 & -54.06875 & 16.45 & 15.65 & 65.69 & $2.42 \pm 0.19$ & $-2.90 \pm 0.10$ & $\ldots$ & $\cdots$ & M \\
\hline DES J033524.96-540230.7 & 53.85400 & -54.04186 & 19.69 & 19.17 & 67.99 & $5.30 \pm 0.57$ & $\ldots$ & $\ldots$ & $\ldots$ & NM \\
\hline DES J033539.04-535556.4 & 53.91267 & -53.93234 & 17.85 & 17.16 & -20.65 & $6.97 \pm 0.26$ & $\ldots$ & $\ldots$ & $\ldots$ & NM \\
\hline DES J033548.04-540349.8 & 53.95017 & -54.06384 & 18.27 & 17.69 & 62.78 & $3.45 \pm 0.22$ & $-2.02 \pm 0.11$ & $\ldots$ & $\ldots$ & M? \\
\hline DES J033603.90-540026.5 & 54.01625 & -54.00737 & 17.79 & 17.09 & -25.16 & $5.87 \pm 0.23$ & $\ldots$ & $\ldots$ & $\ldots$ & NM \\
\hline DES J033623.52-540518.9 & 54.09798 & -54.08857 & 19.75 & 19.23 & 213.51 & $4.33 \pm 0.55$ & $\cdots$ & $\ldots$ & $\ldots$ & NM \\
\hline \multicolumn{11}{|c|}{ GMOS } \\
\hline DES J033539.85-540458.1 & 53.91605 & -54.08281 & 18.33 & 18.59 & 69.30 & $\cdots$ & $\ldots$ & $\ldots$ & $\ldots$ & M \\
\hline
\end{tabular}

Note.

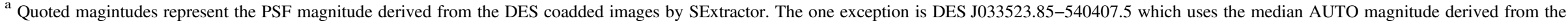
individual DES single epoch images. 
Table 2

Summary of Properties of Reticulum II

\begin{tabular}{|c|c|c|}
\hline Row & Quantity & Value \\
\hline (1) & R.A. (J2000) & $03: 35: 41$ \\
\hline (2) & Decl. (J2000) & $-54: 03: 00$ \\
\hline (3) & Distance (kpc) & 32 \\
\hline (4) & $M_{V, 0}$ & $-3.6 \pm 0.1$ \\
\hline (5) & $L_{V, 0}\left(L_{\odot}\right)$ & $2360 \pm 200$ \\
\hline (6) & $\epsilon$ & $0.60_{-0.20}^{+0.10}$ \\
\hline (7) & $r_{1 / 2}(\mathrm{pc})$ & $55 \pm 5$ \\
\hline (8) & $V_{\text {hel }}\left(\mathrm{km} \mathrm{s}^{-1}\right)$ & $62.8 \pm 0.5$ \\
\hline (9) & $V_{\mathrm{GSR}}\left(\mathrm{km} \mathrm{s}^{-1}\right)$ & $-92.5 \pm 0.5$ \\
\hline$(10)$ & $\sigma\left(\mathrm{km} \mathrm{s}^{-1}\right)$ & $3.3 \pm 0.7$ \\
\hline (11) & $\begin{array}{l}\text { Mass within the half-light } \\
\quad \text { radius }\left(M_{\odot}\right)\end{array}$ & $5.6 \pm 2.4 \times 10^{5}$ \\
\hline$(12)$ & $M_{1 / 2} / L_{V}\left(M_{\odot} / L_{\odot}\right)$ & $470 \pm 210$ \\
\hline (13) & Mean $[\mathrm{Fe} / \mathrm{H}]$ & $-2.65 \pm 0.07$ \\
\hline$(14)$ & Metallicity dispersion (dex) & $0.28 \pm 0.09$ \\
\hline (15) & $\log _{10} J\left(0^{\circ} \cdot 2\right)\left(\mathrm{GeV}^{2} \mathrm{~cm}^{-5}\right)$ & $18.8 \pm 0.6$ \\
\hline$(16)$ & $\log _{10} J(0.5)\left(\mathrm{GeV}^{2} \mathrm{~cm}^{-5}\right)$ & $18.9 \pm 0.6$ \\
\hline
\end{tabular}

Note. Rows (1)-(7) are taken from the DES photometric analysis of Bechtol et al. (2015). Values in rows (8)-(16) are derived in this paper.

obtained by modeling this individual system is larger than is obtained by modeling the entire population of dSphs (Martinez 2015).

Several previously known ultra-faint dwarf galaxies possess larger mean $J$-factors than Ret II, most notably Segue 1, Ursa Major II, and Coma Berenices (Ackermann et al. 2014; Conrad et al. 2015; Geringer-Sameth et al. 2015a). Though the velocity dispersions of Ret II and Segue 1 are consistent within uncertainties, Ret II is more distant $(32 \mathrm{kpc}$ compared to $23 \mathrm{kpc}$ ) and has a larger half-light radius as measured along the major axis (55 pc compared to $29 \mathrm{pc}$ ). The larger distance and larger half-light radius imply a reduced mean $J$-factor relative to Segue 1. In comparison to Ursa Major II, Ret II is at a similar distance, but has a velocity dispersion that is smaller by roughly a factor of two. The larger dispersion, and hence mass, accounts for the larger $J$-factor of Ursa Major II. Coma Berenices is more distant than Ret II (44 kpc compared to $32 \mathrm{kpc}$ ); however, the larger velocity dispersion of Coma Berenices implies a slightly larger mean $J$-factor.

Since Segue 1, Ursa Major II, and Coma Berenices all possess larger $J$-factors than Ret II, we expect dark matter annihilation to produce a larger gamma-ray flux from these objects. However, no gamma-ray excess has been associated with any of the previously known dwarf galaxies (Ackermann et al. 2015). Given comparable gamma-ray sensitivity, it is unlikely that a dark matter annihilation signal would be detected from Ret II without also being detected from dwarf galaxies with higher $J$-factors (Drlica-Wagner et al. 2015; Geringer-Sameth et al. 2015c; Hooper \& Linden 2015).

\section{SUMMARY AND CONCLUSIONS}

We have presented the first spectroscopic analysis of the recently discovered Milky Way satellite Reticulum II. We measure the velocities of 25 Ret II members using high resolution spectroscopy from Magellan/M2FS, as well as metallicities from the CaT lines for 16 members using high resolution spectroscopy from VLT/GIRAFFE and 6 members using low resolution spectroscopy from Gemini South/GMOS. Ret II has a velocity dispersion of $\sigma_{v}=3.3 \pm 0.7 \mathrm{~km} \mathrm{~s}^{-1}$, corresponding to a dynamical mass within its half-light radius of $5.6 \pm 2.4 \times 10^{5} M_{\odot}$ and a mass-to-light ratio of $470 \pm 210 M_{\odot} / L_{\odot}$.

The metallicity of Ret II determined from the CaT is $[\mathrm{Fe} / \mathrm{H}]=-2.65 \pm 0.07$, consistent with that of Segue 1 (Frebel et al. 2014) within the uncertainties, and $\sim 0.2 \mathrm{dex}$ lower than that of any other known galaxy. We find that Ret II has an internal metallicity spread of $0.28 \pm 0.09$ dex, with stars spanning a total range of more than $1 \mathrm{dex}$. Even the most metalrich stars in the galaxy are at $[\mathrm{Fe} / \mathrm{H}] \approx-2.0$, and Ret II contains 4 extremely metal-poor stars with $[\mathrm{Fe} / \mathrm{H}]<-3$. Both its chemical and kinematic properties confirm that Ret II is a dwarf galaxy.

The location of Ret II just $23 \mathrm{kpc}$ away from the LMC suggests that it could have originated as a satellite of the Magellanic system rather than having always been associated with the Milky Way (Bechtol et al. 2015; Koposov et al. 2015). However, our measured systemic radial velocity of $v_{\text {hel }}=$ $62.8 \pm 0.5 \mathrm{~km} \mathrm{~s}^{-1}$ means that Ret II is moving away from the LMC at a minimum velocity of $199 \mathrm{~km} \mathrm{~s}^{-1}$. According to current LMC mass estimates, this velocity likely exceeds the escape velocity of the LMC, indicating that the two objects are not gravitationally bound. This result does not rule out the possibility that Ret II was previously a Magellanic satellite, and future proper motion measurements will shed more light on its origin.

The $J$-factor calculated from the internal kinematics of Ret II is $\log _{10} J=18.8 \pm 0.6 \mathrm{GeV}^{2} \mathrm{~cm}^{-5}$ within a radius of 0.2 , somewhat lower than previously estimated based on the galaxy's distance alone (Drlica-Wagner et al. 2015). The predicted gamma-ray flux from dark matter annihilation in Ret II is therefore likely to be lower than that predicted for several other Milky Way satellites (Drlica-Wagner et al. 2015; Geringer-Sameth et al. 2015c; Hooper \& Linden 2015).

Satellite galaxies like Ret II provide a crucial testing ground for the $\Lambda$ CDM paradigm, and accordingly, the search for ultrafaint galaxies has become a major theme of near-field cosmology. It is expected that many additional Milky Way satellite galaxies could be found in ongoing and near-future wide-field optical imaging surveys (Tollerud et al. 2008; Hargis et al. 2014). The link between newly discovered stellar systems and the dark matter halos in which they may reside is established by follow-up dynamical and chemical analysis. Ret II is the first of several recently reported stellar systems (Bechtol et al. 2015; Kim et al. 2015; Koposov et al. 2015; Laevens et al. 2015; Martin et al. 2015) to be spectroscopically confirmed as a dark-matter-dominated Milky Way satellite galaxy. The spectroscopic campaign to characterize new satellite galaxy candidates represents an essential step in ongoing tests of the standard cosmological model.

This publication is based upon work supported by the National Science Foundation under grant AST-1108811. We thank Dan Kelson for helpful conversations, Anna Frebel for providing the MIKE spectrum of HD 122563, and Becky Canning and Jimmy for helpful conversations on the reduction of VLT spectra. We also thank the anonymous referee for suggestions that improved the presentation of the paper. A.C.R. acknowledges financial support provided by the PAPDRJ. 
CAPES/FAPERJ Fellowship. F.S. acknowledges financial support provided by CAPES under contract No. 3171-13-2. This research has made use of NASA's Astrophysics Data System Bibliographic Services. M2FS was built through partial support via NSF/MRI grant AST-0923160 to Mario Mateo, Ian Thompson and Steven Shectman as co-PIs and its construction was assisted by Jeffrey Crane and John I. Bailey III. The M2FS observations used in this paper were carried out on our behalf by Mario Mateo and Matthew Walker, who were supported in part by NSF grants AST-1312967 (MM) and AST-1313045 (MW). Additional partial operational support of M2FS has been provided by the Department of Astronomy of the University of Michigan and by Carnegie Observatories. Funding for the DES Projects has been provided by the U.S. Department of Energy, the U.S. National Science Foundation, the Ministry of Science and Education of Spain, the Science and Technology Facilities Council of the United Kingdom, the Higher Education Funding Council for England, the National Center for Supercomputing Applications at the University of Illinois at Urbana-Champaign, the Kavli Institute of Cosmological Physics at the University of Chicago, the Center for Cosmology and Astro-Particle Physics at the Ohio State University, the Mitchell Institute for Fundamental Physics and Astronomy at Texas A\&M University, Financiadora de Estudos e Projetos, Fundação Carlos Chagas Filho de Amparo à Pesquisa do Estado do Rio de Janeiro, Conselho Nacional de Desenvolvimento Científico e Tecnológico and the Ministério da Ciência, Tecnologia e Inovação, the Deutsche Forschungsgemeinschaft and the Collaborating Institutions in the Dark Energy Survey. The DES data management system is supported by the National Science Foundation under Grant Number AST-1138766. The DES participants from Spanish institutions are partially supported by MINECO under grants AYA2012-39559, ES2P2013-48274, FPA2013-47986, and Centro de Excelencia Severo Ochoa SEV-2012-0234, some of which include ERDF funds from the European Union. The Collaborating Institutions are Argonne National Laboratory, the University of California at Santa Cruz, the University of Cambridge, Centro de Investigaciones Energéticas, Medioambientales y Tecnológicas-Madrid, the University of Chicago, University College London, the DES-Brazil Consortium, the University of Edinburgh, the Eidgenössische Technische Hochschule (ETH) Zürich, Fermi National Accelerator Laboratory, the University of Illinois at Urbana-Champaign, the Institut de Ciències de l'Espai (IEEC/CSIC), the Institut de Física d'Altes Energies, Lawrence Berkeley National Laboratory, the Ludwig-Maximilians Universität München and the associated Excellence Cluster Universe, the University of Michigan, the National Optical Astronomy Observatory, the University of Nottingham, The Ohio State University, the University of Pennsylvania, the University of Portsmouth, SLAC National Accelerator Laboratory, Stanford University, the University of Sussex, and Texas A\&M University.

Facilities: Magellan: Clay (M2FS), VLT:Kueyen (GIRAFFE), Gemini:South (GMOS).

\section{REFERENCES}

Ackermann, M., Albert, A., Anderson, B., et al. 2014, PhRvD, 89, 042001

Ackermann, M., Albert, A., Anderson, B., et al. 2015, PhRvL, submitted (arXiv:1503.02641)

Adelman-McCarthy, J. K., Agüeros, M. A., Allam, S. S., et al. 2008, ApJS, 175,297
Balbinot, E., Santiago, B. X., Girardi, L., et al. 2015, MNRAS, 449, 1129 Baltz, E., Berenji, B., Bertone, G., et al. 2008, JCAP, 0807, 013

Battaglia, G., \& Starkenburg, E. 2012, A\&A, 539, A123

Bechtol, K., Drlica-Wagner, A., Balbinot, E., et al. 2015, ApJ, 807, 50

Belokurov, V., Zucker, D. B., Evans, N. W., et al. 2007, ApJ, 654, 897 Bergström, L., \& Snellman, H. 1988, PhRvD, 37, 3737

Bertin, E. 2011, in ASP Conf. Ser. 442, Astronomical Data Analysis Software and Systems XX, ed. I. N. Evans et al. (San Francisco, CA: ASP), 435

Brown, T. M., Tumlinson, J., Geha, M., et al. 2014, ApJ, 796, 91

Burgh, E. B., Bershady, M. A., Westfall, K. B., \& Nordsieck, K. H. 2007 , PASP, 119, 1069

Carrera, R., Pancino, E., Gallart, C., \& del Pino, A. 2013, MNRAS, 434, 1681

Cenarro, A. J., Cardiel, N., Gorgas, J., et al. 2001, MNRAS, 326, 959

Chabrier, G. 2001, ApJ, 554, 1274

Charbonnier, A., Combet, C., Daniel, M., et al. 2011, MNRAS, 418, 1526

Chubak, C., Marcy, G., Fischer, D. A., et al. 2012, arXiv:1207.6212

Conrad, J., Cohen-Tanugi, J., \& Strigari, L. E. 2015, arXiv:1503.06348

Desai, S., Armstrong, R., Mohr, J. J., et al. 2012, ApJ, 757, 83

Diehl, H. T., Abbott, T. M. C., Annis, J., et al. 2014, Proc. SPIE, 9149, 91490V

Drlica-Wagner, A., Albert, A., Bechtol, K., et al. 2015, ApJL, submitted (arXiv:1503.02632)

Essig, R., Sehgal, N., \& Strigari, L. E. 2009, PhRvD, 80, 023506

Flaugher, B., Diehl, H. T., Honscheid, K., et al. 2015, AJ, submitted (arXiv: 1504.02900)

Frebel, A., Simon, J. D., Geha, M., \& Willman, B. 2010, ApJ, 708, 560

Frebel, A., Simon, J. D., \& Kirby, E. N. 2014, ApJ, 786, 74

Geringer-Sameth, A., Koushiappas, S. M., \& Walker, M. 2015a, ApJ, 801, 74

Geringer-Sameth, A., Koushiappas, S. M., \& Walker, M. G. 2015b, PhRvD, 91, 083535

Geringer-Sameth, A., Walker, M. G., Koushiappas, S. M., et al. 2015c, PhRvD, submitted (arXiv:1503.02320)

Gilmore, G., Randich, S., Asplund, M., et al. 2012, Msngr, 147, 25

Girardi, L., Groenewegen, M. A. T., Hatziminaoglou, E., \& da Costa, L. 2005, A\&A, 436, 895

Girardi, L., Williams, B. F., Gilbert, K. M., et al. 2010, ApJ, 724, 1030

Gunn, J. E., Lee, B. W., Lerche, I., Schramm, D. N., \& Steigman, G. 1978, ApJ, 223, 1015

Hargis, J. R., Willman, B., \& Peter, A. H. G. 2014, ApJL, 795, L13

Harris, J., \& Zaritsky, D. 2006, AJ, 131, 2514

Hendricks, B., Koch, A., Walker, M., et al. 2014, A\&A, 572, A82

Hook, I. M., Jørgensen, I., Allington-Smith, J. R., et al. 2004, PASP, 116,425

Hooper, D., \& Linden, T. 2015, arXiv:1503.06209

Jordi, K., Grebel, E. K., \& Ammon, K. 2006, A\&A, 460, 339

Kallivayalil, N., van der Marel, R. P., Besla, G., Anderson, J., \& Alcock, C. 2013, ApJ, 764, 161

Kim, D., Jerjen, H., Mackey, D., Da Costa, G. S., \& Milone, A. P. 2015, ApJL, 804, L44

Kirby, E. N., Cohen, J. G., Guhathakurta, P., et al. 2013, ApJ, 779, 102

Kirby, E. N., Lanfranchi, G. A., Simon, J. D., Cohen, J. G., \& Guhathakurta, P. 2011, ApJ, 727, 78

Kirby, E. N., Simon, J. D., Geha, M., Guhathakurta, P., \& Frebel, A. 2008, ApJL, 685, L43

Koch, A., Hansen, T., Feltzing, S., \& Wilkinson, M. I. 2014, ApJ, 780, 91

Koch, A., McWilliam, A., Grebel, E. K., Zucker, D. B., \& Belokurov, V. 2008 , ApJL, 688, L13

Koposov, S. E., Belokurov, V., Torrealba, G., \& Evans, N. W. 2015, ApJ, 805,130

Koposov, S. E., Gilmore, G., Walker, M. G., et al. 2011, ApJ, 736, 146 Laevens, B. P. M., Martin, N. F., Ibata, R. A., et al. 2015, ApJL, 802, L18

Lee, Y. S., Beers, T. C., Sivarani, T., et al. 2008, AJ, 136, 2022

Marigo, P., Girardi, L., Bressan, A., et al. 2008, A\&A, 482, 883

Martin, N. F., Ibata, R. A., Chapman, S. C., Irwin, M., \& Lewis, G. F. 2007, MNRAS, 380, 281

Martin, N. F., Nidever, D. L., Besla, G., et al. 2015, ApJL, 804, L5

Martinez, G. D. 2015, MNRAS, 451, 2524

Martinez, G. D., Minor, Q. E., Bullock, J., et al. 2011, ApJ, 738, 55

Massey, P., \& Hanson, M. M. 2013, Astronomical Spectroscopy, ed. T. D. Oswalt \& H. E. Bond (Dordrecht, Netherlands: Springer), 35

Mateo, M., Bailey, J. I., Crane, J., et al. 2012, Proc. SPIE, 8446, 84464Y

McConnachie, A. W. 2012, AJ, 144, 4

Mohr, J. J., Armstrong, R., Bertin, E., et al. 2012, Proc. SPIE, 8451, 84510D

Navarro, J. F., Frenk, C. S., \& White, S. D. 1997, ApJ, 490, 493

Newman, J. A., Cooper, M. C., Davis, M., et al. 2013, ApJS, 208, 5

Nidever, D. L., Majewski, S. R., Muñoz, R. R., et al. 2011, ApJL, 733, L10 
Norris, J. E., Wyse, R. F. G., Gilmore, G., et al. 2010, ApJ, 723, 1632

Pasquini, L., Avila, G., Allaert, E., et al. 2000, Proc. SPIE, 4008, 129

Robin, A. C., Reylé, C., Derrière, S., \& Picaud, S. 2003, A\&A, 409, 523

Rozo, E., Rykoff, E. S., Koester, B. P., et al. 2009, ApJ, 703, 601

Schlafly, E. F., \& Finkbeiner, D. P. 2011, ApJ, 737, 103

Sevilla, I., Armstrong, R., Bertin, E., et al. 2011, arXiv:1109.6741

Simon, J. D., \& Geha, M. 2007, ApJ, 670, 313

Simon, J. D., Geha, M., Minor, Q. E., et al. 2011, ApJ, 733, 46

Strigari, L. E., Koushiappas, S. M., Bullock, J. S., \& Kaplinghat, M. 2007, PhRvD, 75, 083526

Strigari, L. E., Koushiappas, S. M., Bullock, J. S., et al. 2008, ApJ, 678, 614

Tollerud, E. J., Bullock, J. S., Strigari, L. E., \& Willman, B. 2008, ApJ, 688,277
Udry, S., Mayor, M., Maurice, E., et al. 1999, in ASP Conf. Ser. 185, IAU Coll. 170, Precise Stellar Radial Velocities, ed. J. B. Hearnshaw \& C. D. Scarfe (San Francisco, CA: ASP), 383

van Dokkum, P. G. 2001, PASP, 113, 1420

Vanhollebeke, E., Groenewegen, M. A. T., \& Girardi, L. 2009, A\&A, 498, 95

Vargas, L. C., Geha, M., Kirby, E. N., \& Simon, J. D. 2013, ApJ, 767, 134

Walker, M. G., Mateo, M., Olszewski, E. W., et al. 2006, AJ, 131, 2114

Weisz, D. R., Dolphin, A. E., Skillman, E. D., et al. 2014a, ApJ, 789, 147

Weisz, D. R., Johnson, B. D., \& Conroy, C. 2014b, ApJL, 794, L3

Willman, B., Dalcanton, J. J., Martinez-Delgado, D., et al. 2005, ApJL, 626, L85

Wolf, J., Martinez, G. D., Bullock, J. S., et al. 2010, MNRAS, 406, 1220

Zucker, D. B., Belokurov, V., Evans, N. W., et al. 2006, ApJL, 650, L41 